\title{
Quantum master equations for entangled qubit environments
}

\author{
Shakib Daryanoosh, ${ }^{1, *}$ Ben Q. Baragiola, ${ }^{2,1}$ Thomas Guff, ${ }^{1}$ and Alexei Gilchrist ${ }^{1}$ \\ ${ }^{1}$ Centre for Engineered Quantum Systems, Department of Physics and Astronomy, Macquarie University, Sydney, NSW 2122, Australia \\ ${ }^{2}$ Centre for Quantum Computation and Communication Technology, School of Science, RMIT University, Melbourne, Victoria 3001, Australia
}

(Received 11 July 2018; published 5 December 2018)

\begin{abstract}
We study the Markovian dynamics of a collection of $n$ quantum systems coupled to an irreversible environmental channel consisting of a stream of entangled qubits. Within the framework of repeated quantum interactions, we derive the master equation for the joint-state dynamics of the $n$ quantum systems. We investigate the evolution of the joint state for two-qubit environments where the presence of antidiagonal coherences in the state of the bath qubits (in the local energy basis) is essential for preserving and generating entanglement between two remote quantum systems. However, maximally entangled bath qubits, such as Bell states, exhibit exceptional behavior, where the master equation does not have a unique steady state and can destroy entanglement between the systems. For the general case of $n$-qubit environments we show that antidiagonal coherences that arise from multibody entanglement in the bath qubits do not affect the composite system evolution in the weak-coupling regime.
\end{abstract}

DOI: 10.1103/PhysRevA.98.062104

\section{INTRODUCTION}

Open quantum systems are the subject of extensive research since physical quantum systems cannot be entirely isolated from their surroundings. The influence of the environment often manifests as unwanted noise that can thwart attempts to exploit intrinsic quantum properties for quantum computing, communication, and metrology [1]. Open systems tend to lose their key quantum properties-coherence and entanglement - as they interact with the environment. This is not inevitable, however, and much research has focused on engineered environments [2] for various tasks including quantum computing [3-5] and the generation of novel steady states [6-10]. This can be achieved through a combination of precise structuring of system-bath interactions and preparation of the environment in particular states.

One can take many approaches to the description of open quantum systems. In standard quantum optical treatments, the electromagnetic field serves as the environment [11-13], and the dynamics of the reduced quantum states is given by a master equation (ME) after the environmental degrees of freedom are traced out. An alternative approach is that of repeated quantum interactions [14-17], also called collision models [18-22], which treats the system-environment coupling discretely. The environment is comprised of a chain of identical and independent quantum ancillae which sequentially couple to the system and are then traced out. Taking a continuous limit [15,16,20-26], the resulting dynamical map on the reduced system state becomes such that it is described by a Lindblad ME. This formalism has been applied in the context of quantum thermodynamics [27-31], as well as quantum optics and information [32], and has experimentally been tested using free-space optics [33]. The framework of

\footnotetext{
*sh.daryanoosh@gmail.com
}

repeated quantum interactions has also proven useful for the study of correlated quantum channels [20,34,35]. When the ancillae are correlated, the reduced-state dynamics can exhibit non-Markovian behavior [21,36,37], such as for propagating bath states with a fixed number of distributed excitations [38].

In this article we consider a correlated environment that interacts with many quantum systems simultaneously. The environment consists of a stream of $n$ ancilla qubits, each coupled to its own system. The $n$ qubits arrive entangled with one another at each interaction time, but they are not entangled across different times, which allows us to derive a Lindblad ME for the joint state of the quantum systems. Depending on the state of the qubits, the ME can generate nontrivial dynamics of the reduced systems. This provides the tools to tackle the problem of converting coherences and/or entanglement in the environment into quantum correlations in the system [39-43]. Accordingly, we analyze in detail the pedagogical case of $n=2$ qubits in the bath, which can be prepared as a stream of entangled states, as it provides the canonical method for transferring qubit entanglement to system entanglement. When the bath is prepared arbitrarily close to a maximally entangled state, the system is driven to an entangled pure state, such as a two-mode squeezed state for the case of two optical cavities. Surprisingly, if the qubit bath is exactly in a Bell state, the system fails to converge to a unique steady state. For $n$-qubit baths $(n>2)$, we find that for certain multiqubit entangled environments such as baths prepared in $X$ states (which have nonzero elements only on the diagonal and antidiagonal entries of the state matrix when expressed in the local energy basis) [44,45], the bath entanglement cannot be transferred to the systems, as a direct consequence of the weak-coupling limit

The manuscript is organized as follows. In Sec. II the underlying framework behind our analysis is explained. We first present and interpret the two-qubit bath ME in Sec. III. We give two forms for the ME, which are useful for pure-state 
and general mixed-state baths, respectively. In Sec. V and Sec. IV we apply the formalism to study the dynamics and steady states for two sets of quantum system: optical cavities and two-level atoms. The general case of an $n$-qubit bath is dealt with in Sec. VI. Finally, in Sec. VII we summarize the findings of this paper and propose future directions.

\section{REPEATED INTERACTION WITH A BATH OF $\boldsymbol{n}$ ENTANGLED QUBITS}

We derive dynamical maps and master equations within the structure of repeated quantum interactions [14,17,30,32]. In this formalism, a quantum system in Hilbert space $\mathscr{H}_{S}$ couples to an environment which is comprised of a stream of identical and independent quantum systems such that $\mathscr{H}_{E} \equiv \bigotimes_{k} \mathscr{H}_{E}^{(k)}$. We assume the environment has infinitely many elements, although in principle it can be finite. Each environmental element sequentially interacts with the system over a short time interval of duration $\Delta t=t_{k+1}-t_{k}$ after which it no longer interacts with the system. Tracing over the environmental degrees of freedom yields a map on the system of interest in $\mathscr{H}_{S}$. This is similar to the standard scenario for open systems in quantum optics where a bosonic probe field interacts with the system in a continuous-in-time manner [12]. However, the situation here is different in two ways. First, the system-environment coupling is fundamentally discrete, although we will ultimately consider short-time interactions and take a continuous limit $[15,16,46]$. Second, the environmental systems are qubits rather than bosonic modes. This serves not only to model physical situations where streams of qubits interact with a fixed quantum system [47], but in addition the results fit into the framework of quantum computing and simulation [32].

For each interaction time interval $\Delta t$ the total Hilbert space of the system plus the segment of the environment interacting at that time is $\mathscr{H}=\mathscr{H}_{S} \otimes \mathscr{H}_{E}^{(k)}$, and the Hamiltonian is

$$
\hat{H}=\hat{H}_{S}+\hat{H}_{E}^{(k)}+\hat{H}_{S E}^{(k)},
$$

corresponding to the bare Hamiltonians of the system $\hat{H}_{S}$ and of the environment $\hat{H}_{E}^{(k)}$, and the system-environment interaction, $\hat{H}_{S E}^{(k)}$. Here, we consider each environmental piece, labeled by $k$, as a set of $n$ entangled qubits. Each of the $n$ qubits couples to its own quantum system; see Fig. 1 for the case of $n=2$. That is, in each time interval $\Delta t, n$ entangled qubits interact with $n$ quantum systems, which themselves are left arbitrary and can in general be remote from each other. Note that the bath qubits within a single time interval are entangled, but they are not entangled between time intervals. This type of environmental entanglement drives non-Markovian dynamics $[34,38,48-51]$ and will be treated separately.

Each subsystem interacts with its respective qubit via a coupling operator $\hat{c}_{j}$. The bare and interaction Hamiltonians are

$$
\begin{gathered}
\hat{H}_{S}=\sum_{\ell=1}^{n} \omega_{S_{\ell}} \hat{c}_{\ell}^{\dagger} \hat{c}_{\ell}, \quad \hat{H}_{E}^{(k)}=\sum_{\ell=1}^{n} \omega_{E_{\ell}} \hat{\sigma}_{\ell}^{\dagger} \hat{\sigma}_{\ell}, \\
\hat{H}_{S E}^{(k)}=\sum_{\ell=1}^{n} \lambda_{\ell}\left(\hat{c}_{\ell} \hat{\sigma}_{\ell}^{\dagger}+\hat{c}_{\ell}^{\dagger} \hat{\sigma}_{\ell}\right),
\end{gathered}
$$
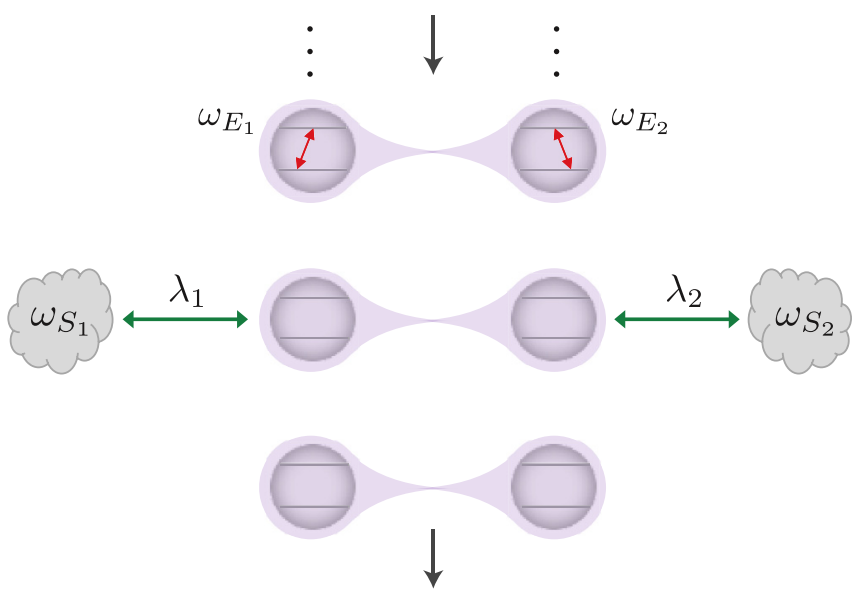

FIG. 1. Conceptual diagram of the physical model where a stream of entangled qubits sequentially interacts with separate quantum systems. Shown here is the case of $n=2$ qubits.

where $\omega_{\ell}$ and $\omega_{E_{\ell}}$ are the respective transition frequencies of the subsystems and the bath qubits, $\lambda_{\ell}$ is the coupling strength between the $\ell$ th subsystem and its qubit, and the bath qubit lowering operator is $\hat{\sigma}_{\ell}=|\mathrm{g}\rangle_{\ell}\langle\mathrm{e}|$. Here, it is assumed that $\hat{c}_{\ell}$ are eigenoperators of the system Hamiltonian [52]. In the interaction picture with respect to the bare Hamiltonian, $\hat{H}_{S}+\hat{H}_{E}^{(k)}$, the joint unitary evolution is generated by the time-dependent Hamiltonian,

$$
\hat{H}_{I}^{(k)}=\sum_{\ell=1}^{n} \lambda_{\ell}\left(\hat{c}_{\ell}^{\dagger} \hat{\sigma}_{\ell} e^{-i \delta_{\ell} t}+\text { H.c. }\right)
$$

where $\delta_{\ell}:=\omega_{E_{\ell}}-\omega_{S_{\ell}}$ is the detuning and H.c. stands for Hermitian conjugate. The detuning is included here for completeness; henceforth, we focus our attention on resonant system-qubit interactions, $\delta_{\ell}=0$, which gives $\left[\hat{H}_{S}+\hat{H}_{E}^{(k)}, \hat{H}_{S E}^{(k)}\right]=0$. That is, the resonant interaction is a thermal process where any energy lost by the bath is absorbed by the subsystems and vice versa [53]. One models a specific reservoir by selecting a particular state for the environmental qubits. Investigating situations where the bath qubits are entangled is the focus of this article.

A dynamical map for the joint state of the $n$ fixed quantum systems is found by tracing out the the environmental qubits after the interaction, $\hat{U}_{I}^{(k)}=\exp \left(-i \hat{H}_{I}^{(k)} \Delta t\right)$, generated by the time-independent Hamiltonian in Eq. (4):

$$
\hat{U}_{I}^{(k)}=\hat{\mathbb{1}}-i \hat{H}_{I}^{(k)} \Delta t-\frac{1}{2}\left(\hat{H}_{I}^{(k)}\right)^{2} \Delta t^{2}+O\left(\Delta t^{3}\right),
$$

where in the weak-coupling regime the unitary time-evolution operator is expanded up to second order in $\Delta t$. At each time interval the incoming bath qubits and the quantum systems are assumed to be in a product state, so the dynamical map is completely positive and trace preserving. Assuming that only one qubit interacts with each system in an interaction time and the joint state of the $n$-qubit environment interacting in the time interval is $\hat{\rho}_{E}$, the dynamical map for a single time step $\Delta t$ is given by

$$
\mathcal{M}(\Delta t) \hat{\rho}\left(t_{k}\right):=\operatorname{Tr}_{E}\left[\hat{U}_{I}^{(k)}\left(\hat{\rho}\left(t_{k}\right) \otimes \hat{\rho}_{E}^{(k)}\right) \hat{U}_{I}^{(k) \dagger}\right] .
$$


The fact that each set of $n$ qubits, described by $\hat{\rho}_{E}$, is independent from other sets means that this dynamical map is explicitly Markovian (i.e., arises from a memoryless environment). This is because the joint state of the input environment is a tensor product state across interaction time intervals, $\hat{\rho}_{E} \equiv$ $\bigotimes_{k} \hat{\rho}_{E}^{(k)}$ [50]. Thus the dynamical map at every subsequent time interval is of the form of Eq. (6), using the system state from the previous time interval and a fresh environmental state $\hat{\rho}_{E}$.

Rather than using the discrete dynamical maps in Eq. (6), we take a continuous-time limit and describe the reduced-state dynamics by a Markovian ME. A master equation can be derived from Eq. (6) under a set of standard assumptions. First, each environmental qubit spends the same amount of time $\Delta t$ interacting with its local system. Second, the Markov condition requires that the $n$-qubit environment in each time interval is independent of other intervals. Third, the systembath coupling is weak $\lambda_{\ell}<\omega_{S_{\ell}}$, while $\lambda_{\ell} \Delta t \ll 1$. Finally, we make a typical assumption that $\operatorname{Tr}\left[\hat{O}_{E} \hat{\rho}_{E}\right]=0$, which indicates that the averages of those environment operators $\hat{O}_{E}$, which appear in the interaction Hamiltonian $\hat{H}_{\mathrm{SE}}^{(k)}$, over the environment state vanish. This allows us to construct a ME including only dissipator terms with no unitary evolution. See Appendix A for the details of derivations.

One might be able to take different approaches depending on the problem under investigation either by deriving a coarsegrained master equation or finding an interpolating scheme for any time between two discrete times $t_{k}$ and $t_{k+1}$ which requires error analysis [23-25] or taking the system-environment coupling strengths to infinity $\lambda_{\ell} \rightarrow \infty$ at $\Delta t \rightarrow 0[20,22,26,28]$. We also refer an interested reader to Refs. [15-17] for more rigorous mathematical analyses about continuous repeated quantum interactions.

It is noted that the interaction Hamiltonian that arises naturally in collision models, Eq. (4), has the form typically associated with the rotating-wave approximation employed in quantum optics.

From now on, for the ease of notation we drop the explicit superscripts for the system and environment state unless confusion could arise.

\section{MASTER EQUATIONS FOR TWO-QUBIT BATHS}

In this section we focus on two-qubit baths as the quintessential extension of the single-qubit baths that are typically studied [28,30-32,38]. Two-qubit baths can exhibit nonclassical correlations including maximal entanglement. We investigate how two-qubit baths modify correlations between two remote subsystems - optical cavities in Sec. IV and two-level atoms in Sec. V.

Let us assume that in each time interval the two bath qubits are prepared in the state

$$
\hat{\rho}_{E}=\sum_{l}\left|\psi_{E_{l}}\right\rangle\left\langle\psi_{E_{l}}\right|
$$

where

$$
\begin{aligned}
& \left|\psi_{E_{1}}\right\rangle=b_{\mathrm{ee}}|\mathrm{ee}\rangle+b_{\mathrm{gg}}|\mathrm{gg}\rangle, \\
& \left|\psi_{E_{2}}\right\rangle=b_{\mathrm{ge}}|\mathrm{ge}\rangle+b_{\mathrm{eg}}|\mathrm{eg}\rangle,
\end{aligned}
$$

and the coefficients satisfy

$$
b_{j k} \in \mathbb{C}, \quad \sum_{j \in\{\mathrm{e}, \mathrm{g}\}}\left|b_{j j}\right|^{2}=\sum_{j \neq k}\left|b_{j k}\right|^{2}=1 .
$$

We insert Eq. (5a) into the discrete dynamical map for the reduced system state, Eq. (6), and then evaluate the terms under the two-qubit bath state in Eq. (8). It is then straightforward to obtain the Markovian ME whose details can be found in Appendix A, yields the following master equation $(\hbar=1)$, for the reduced state $\hat{\rho}$ :

$$
\dot{\hat{\rho}}(t)=\sum_{m=1}^{4} \mathcal{D}\left[\hat{L}_{m}\right] \hat{\rho},
$$

where the jump operators are

$$
\begin{aligned}
& \hat{L}_{1}=\sqrt{\gamma_{1}} b_{\mathrm{gg}} \hat{c}_{1}+\sqrt{\gamma_{2}} b_{\mathrm{ee}} \hat{c}_{2}^{\dagger}, \\
& \hat{L}_{2}=\sqrt{\gamma_{1}} b_{\mathrm{ee}} \hat{c}_{1}^{\dagger}+\sqrt{\gamma_{2}} b_{\mathrm{gg}} \hat{c}_{2}, \\
& \hat{L}_{3}=\sqrt{\gamma_{1}} b_{\mathrm{ge}} \hat{c}_{1}+\sqrt{\gamma_{2}} b_{\mathrm{eg}} \hat{c}_{2}, \\
& \hat{L}_{4}=\sqrt{\gamma_{1}} b_{\mathrm{eg}} \hat{c}_{1}^{\dagger}+\sqrt{\gamma_{2}} b_{\mathrm{ge}} \hat{c}_{2}^{\dagger},
\end{aligned}
$$

and the Lindblad superoperator is defined as

$$
\mathcal{D}[\hat{o}] \hat{\rho}=\hat{o} \hat{\rho} \hat{o}^{\dagger}-\frac{1}{2}\left\{\hat{o}^{\dagger} \hat{o}, \hat{\rho}\right\}_{+},
$$

with $\{\hat{A}, \hat{B}\}_{+}=\hat{A} \hat{B}+\hat{B} \hat{A}$. The relative rates are given by $\gamma_{\ell}=\lambda_{\ell}^{2} \Delta t$.

The master equation generates incoherent dynamics in the reduced system state $\hat{\rho}(t)$. The quantum system undergoes correlated dissipation as described by the jump operators in Eq. (11). Each jump operator drives a dissipative process given by combinations of loss $\left(\hat{c}_{\ell}\right)$ and heating $\left(\hat{c}_{\ell}^{\dagger}\right)$ across subsystems 1 and 2 .

Interestingly, the jump operators (11) are determined by the state in two two-dimensional subspaces of the qubit bath, spanned by either $\{|g g\rangle,|e e\rangle\}$ or $\{|g e\rangle,|e g\rangle\}$. Equivalently, each subspace is spanned by two Bell states. Populations and coherences within subspace $\{|g g\rangle$, |ee $\rangle\}$ contribute to jump operators $\hat{L}_{1}$ and $\hat{L}_{2}$, and similarly populations and coherences within subspace $\{\mid$ ge $\rangle, \mid$ eg $\rangle\}$ contribute to jump operators $\hat{L}_{3}$ and $\hat{L}_{4}$. Coupling between the subspaces is due to certain single-qubit coherences.

\section{Alternative form for the master equation}

We transform the master equation into another useful form by expanding Eq. (10) and collecting terms according to coefficients,

$$
\begin{aligned}
\dot{\hat{\rho}}(t)= & \sum_{\ell=1}^{2} \gamma_{\downarrow, \ell} \mathcal{D}\left[\hat{c}_{\ell}\right] \hat{\rho}+\sum_{\ell=1}^{2} \gamma_{\uparrow, \ell} \mathcal{D}\left[\hat{c}_{\ell}^{\dagger}\right] \hat{\rho} \\
& +\gamma_{\downarrow \downarrow} \mathcal{S}\left[\hat{c}_{1}, \hat{c}_{2}\right] \hat{\rho}+\gamma_{\downarrow \downarrow}^{*} \mathcal{S}\left[\hat{c}_{1}^{\dagger}, \hat{c}_{2}^{\dagger}\right] \hat{\rho} \\
& +\gamma_{\downarrow \uparrow} \mathcal{S}\left[\hat{c}_{1}, \hat{c}_{2}^{\dagger}\right] \hat{\rho}+\gamma_{\downarrow \uparrow}^{*} \mathcal{S}\left[\hat{c}_{1}^{\dagger}, \hat{c}_{2}\right] \hat{\rho},
\end{aligned}
$$

where we have defined a superoperator,

$$
\mathcal{S}\left[\hat{o}_{1}, \hat{o}_{2}\right] \hat{\rho}:=\hat{o}_{1} \hat{\rho} \hat{o}_{2}+\hat{o}_{2} \hat{\rho} \hat{o}_{1}-\frac{1}{2}\left\{\hat{o}_{1} \hat{o}_{2}+\hat{o}_{2} \hat{o}_{1}, \hat{\rho}\right\}_{+},
$$


that is symmetric in the arguments. The coefficients in Eq. (13) are given by

$$
\begin{aligned}
& \gamma_{\downarrow, 1}=\gamma_{1}\left(\left|b_{\mathrm{gg}}\right|^{2}+\left|b_{\mathrm{ge}}\right|^{2}\right), \\
& \gamma_{\uparrow, 1}=\gamma_{1}\left(\left|b_{\mathrm{ee}}\right|^{2}+\left|b_{\mathrm{eg}}\right|^{2}\right), \\
& \gamma_{\downarrow, 2}=\gamma_{2}\left(\left|b_{\mathrm{gg}}\right|^{2}+\left|b_{\mathrm{eg}}\right|^{2}\right), \\
& \gamma_{\uparrow, 2}=\gamma_{2}\left(\left|b_{\mathrm{ee}}\right|^{2}+\left|b_{\mathrm{ge}}\right|^{2}\right), \\
& \gamma_{\downarrow \downarrow}=\sqrt{\gamma_{1} \gamma_{2}} b_{\mathrm{gg}} b_{\mathrm{ee}}^{*}, \\
& \gamma_{\downarrow \uparrow}=\sqrt{\gamma_{1} \gamma_{2}} b_{\mathrm{ge}} b_{\mathrm{eg}}^{*} .
\end{aligned}
$$

The first four coefficients in Eqs. (15a)-(15d) are positive and can be interpreted as rates. The final two Eqs. (15e) and (15f) are complex and the superoperator terms that they multiply may in general interfere with other terms including the local dissipators. This is indeed a consequence of Eq. (13) not being in diagonal form with respect to the jump operators. The MEs in Eq. (10) and Eq. (13) are identical, but each may be more useful for certain calculations.

One can get some insight about the system dynamics by parameterizing the state of the environment in an alternate form

$$
\hat{\rho}_{E}=\sum_{l} p_{l} \hat{\rho}_{E_{1}^{(l)}} \otimes \hat{\rho}_{E_{2}^{(l)}}+\mathcal{C},
$$

where $\sum_{l} p_{l}=1$. The first term represents a convex sum of some product states for each qubit in the bath and the correlation term is

$$
\left.\mathcal{C}=b_{\text {ee }} b_{\text {gg }}^{*} \mid \text { ee }\right\rangle\left\langle\text { gg }\left|+b_{\text {eg }} b_{\text {ge }}^{*}\right| \text { eg }\right\rangle\langle\text { ge }|+\text { H.c. }
$$

This representation of the environment's state sheds more light on the role of bipartite correlations in entangling the subsystems. That is to say, recalling Eqs. (16), (17), (13), and (15), it can be seen that entanglement in the bath does not play any role in the local dissipation processes described by the first two terms in the ME. However, it does show up in the two-excitation processes via the complex coefficients, Eqs. (15e) and (15f). This indicates that any nonlocal jumps are due to the presence of bipartite quantum correlations in the bath.

\section{TWO REMOTE CAVITIES: TWO-MODE SQUEEZING}

A stream of qubits interacting with a harmonic oscillator is the prototype for a variety of tasks. For example, twolevel Rydberg atoms interacting with an ultra-high-finesse microwave cavity have been used for quantum nondemolition measurements of photon number [47] and stabilization of Fock states in the cavity [54]. We consider here the natural extension of this system to two remote cavities [41,42,55,56]. This could be realized, for example, by directing two separate, independently prepared streams of atoms close to one another in a laser field tuned near a Rydberg transition. The Rydberg blockade entangles each successive pair of atoms [57,58], one from each stream, which are then sent to separate cavities.

The subsystems are single-mode cavities with creation and annihilation operators $\hat{a}_{\ell}$ and $\hat{a}_{\ell}^{\dagger}(\ell=1,2)$ that satisfy canonical commutation relations, $\left[\hat{a}_{\ell}, \hat{a}_{m}^{\dagger}\right]=\delta_{\ell, m}$. We consider an arbitrary two-qubit bath state in the two-dimensional subspace spanned by $\{|\mathrm{gg}\rangle, \mid$ ee $\rangle\}$, expressed as $\left|\psi_{E}\right\rangle=\left|\psi_{E_{1}}\right\rangle$. That is, the coefficients in Eq. (8) take values $b_{\text {eg }}=b_{\mathrm{ge}}=0$, while $\left|b_{\mathrm{gg}}\right|^{2}+\left|b_{\mathrm{ee}}\right|^{2}=1$. For simplicity each cavity couples to its respective stream of bath qubits with the same rate $\left(\lambda_{1}=\lambda_{2}\right)$ via an interaction that exchanges excitations, which corresponds to $\hat{c}_{\ell} \rightarrow \hat{a}_{\ell}$. Thus the ME in Eq. (10) has jump operators $\hat{L}_{3}=\hat{L}_{4}=0$ and

$$
\begin{aligned}
& \hat{L}_{1}=\sqrt{\gamma}\left(b_{\mathrm{gg}} \hat{a}_{1}+b_{\mathrm{ee}} \hat{a}_{2}^{\dagger}\right), \\
& \hat{L}_{2}=\sqrt{\gamma}\left(b_{\mathrm{gg}} \hat{a}_{2}+b_{\mathrm{ee}} \hat{a}_{1}^{\dagger}\right),
\end{aligned}
$$

reminiscent of two-mode squeezing transformation.

The connection to two-mode squeezing can be made explicit under certain conditions. When $\left|b_{\text {gg }}\right|>1 / \sqrt{2}$ we can define a strictly positive effective rate given by the population difference,

$$
\Gamma:=\gamma\left(\left|b_{\mathrm{gg}}\right|^{2}-\left|b_{\mathrm{ee}}\right|^{2}\right) .
$$

Then, the jump operators can be rewritten as

$$
\begin{aligned}
& \hat{L}_{1}=\sqrt{\Gamma}\left[\cosh (r) \hat{a}_{1}+e^{i \vartheta} \sinh (r) \hat{a}_{2}^{\dagger}\right], \\
& \hat{L}_{2}=\sqrt{\Gamma}\left[\cosh (r) \hat{a}_{2}+e^{i \vartheta} \sinh (r) \hat{a}_{1}^{\dagger}\right],
\end{aligned}
$$

where the squeezing amplitude $r$ is related to the coefficients via the relations,

$$
\cosh (r)=\frac{\left|b_{\mathrm{gg}}\right|}{\sqrt{\left|b_{\mathrm{gg}}\right|^{2}-\left|b_{\mathrm{ee}}\right|^{2}}},
$$

and the squeezing angle $\vartheta$ is given by the phase of $b_{\text {ee }}$ relative to $b_{\mathrm{gg}}$. The jump operators are explicitly given by a two-mode squeezing transformation on the cavity annihilation operators, $\hat{L}_{\ell}=\sqrt{\Gamma} \hat{S} \hat{a}_{\ell} \hat{S}^{\dagger}$, where the unitary, two-mode squeezing operator is $[59,60]$

$$
\hat{S}=e^{\zeta^{*} \hat{a}_{1} \hat{a}_{2}-\zeta \hat{a}_{1}^{\dagger} \hat{a}_{2}^{\dagger},}
$$

with complex squeezing parameter $\zeta=r e^{i \vartheta}$. The transformation is in the Schrödinger picture because the jump operators are nullifiers $[61,62]$ of the two-mode squeezed vacuum state. This can be seen directly by transforming the action of the annihilation operators,

$$
\hat{a}_{\ell}|0\rangle_{2}=0 \rightarrow \hat{S} \hat{a}_{\ell} \hat{S}^{\dagger} \hat{S}|0\rangle_{2} \propto \hat{L}_{\ell}|r, \vartheta\rangle_{2}=0,
$$

where the two-mode squeezed vacuum state is

$$
|r, \vartheta\rangle_{2}:=\hat{S}|0\rangle_{2}
$$

Thus the steady state of the ME is a pure, Gaussian, twomode squeezed vacuum state $|r, \vartheta\rangle_{2}$ with squeezing that depends on the qubit-bath coefficients. Preparing the qubit bath in a maximally entangled state, $\left|b_{\text {gg }}\right|=1 / 2$, is the limit of infinite squeezing, $r \rightarrow \infty$. The dynamical preparation of $|r, \vartheta\rangle_{2}$ from a two-mode vacuum state is shown for various values of the squeezing parameter $r$ in Fig. 2(a). The state is dissipatively cooled via interaction with the two-qubit bath towards the steady state; details can be found in Appendix B. We quantify the approach to $|r, \vartheta\rangle_{2}$ with the Uhlmann-Jozsa fidelity, which can be calculated from the covariance matrix; see Eq. (B13). As $r$ is increased, the effective rate $\Gamma$ decreases according to Eq. (19). That the time to approach the steady 

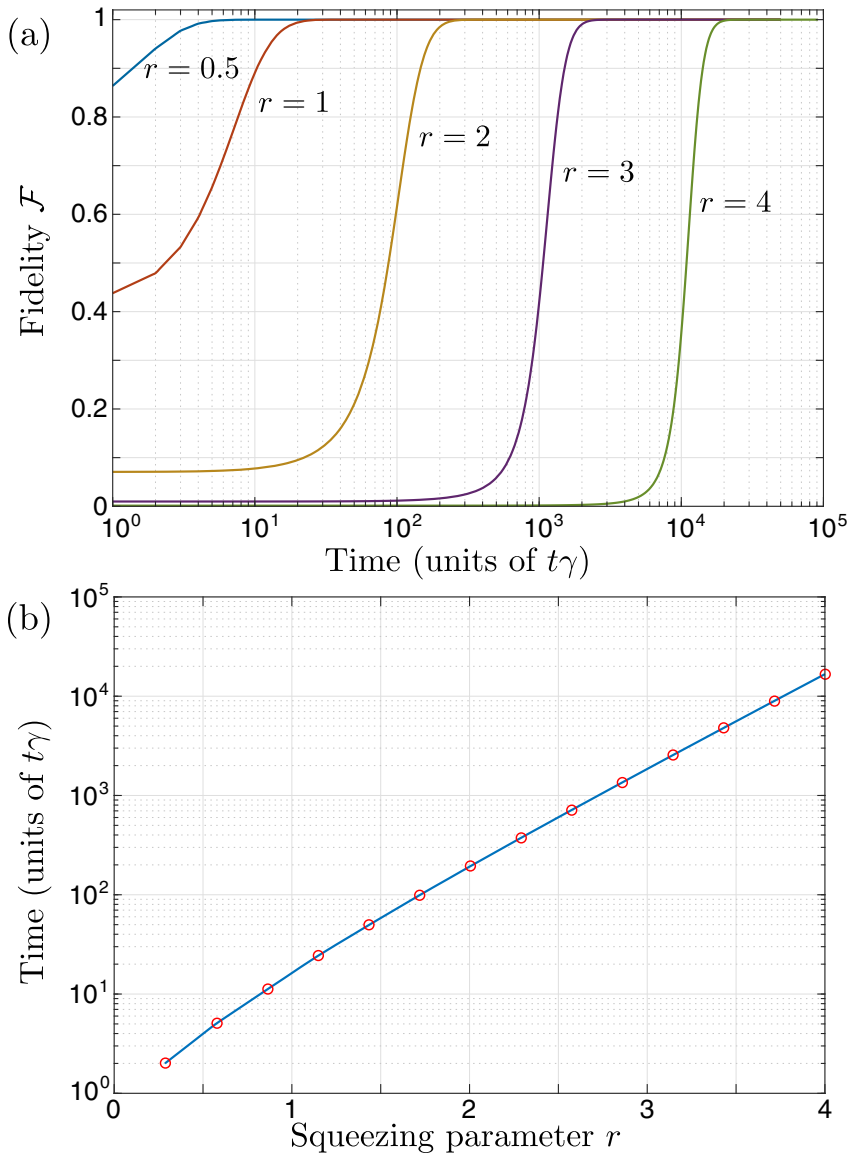

FIG. 2. Dissipative preparation of a two-mode squeezed vacuum state across two remote cavities. The joint cavity state, initially prepared in two-mode vacuum, evolves under the ME in Eq. (10) with jump operators given by Eq. (18). The associated squeezing parameter $r$ is related to the bath qubit coefficients; see Eq. (21). For the plotted values of $r$, the ground-state coefficient magnitudes are $\left|b_{\text {gg }}\right| \approx\{0.908,0.796,0.720,0.709,0.707\}$ and the squeezing angle is set to $\vartheta=0$. Smaller $\left|b_{\mathrm{gg}}\right|$ corresponds to larger squeezing and infinite squeezing to $\left|b_{\mathrm{gg}}\right|=1 / \sqrt{2}$. (a) Fidelity, Eq. (B13), of the state with the two-mode squeezed vacuum state Eq. (24) for various values of $r$. (b) Time at which the fidelity $\mathcal{F}$ surpasses 0.98 as a function of the squeezing parameter $r$.

state $|r, \vartheta\rangle_{2}$ increases exponentially with the squeezing parameter $r$-see Fig. 2(b)-is unsurprising, since more highly squeezed states contain more energy.

Typical optical squeezing protocols are limited by small nonlinearities in parametric down conversion or lightmatter interactions [60] with achieved state-of-the-art optical two-mode squeezing $\sim 10 \mathrm{~dB}$ [63-65], where no. $\mathrm{dB}=$ $10 \log _{10}\left(e^{2 r}\right)$. Here, efficient preparation of bath qubits in near-maximally entangled states can be leveraged to generate and stabilize extremely high level two-mode squeezing; for example, $r=3$ corresponds to $\sim 26 \mathrm{~dB}$. Several recent protocols take a similar tack to the one we present here. A proposal to entangle remote superconducting qubits in separate cavities involves generating two-mode squeezed states using reservoir engineering via a commonly coupled three-wave mixer [56]. The jump operators in their ME are identical to those in Eq. (20). Three-wave mixing has also been used to demonstrate production of two-mode squeezed states in spatially separated microwave transmission lines [66]. Finally, while our focus has been on two-mode squeezing, we note that stabilization of single-mode squeezed states in a cavity using entangled qubit pairs that arrive in succession has been proposed [67].

In the opposite regime, where $\left|b_{\mathrm{gg}}\right|<1 / \sqrt{2}$, there is no unitary Bogoliubov transformation that transforms the operators $\hat{a}_{j}$ while maintaining the canonical commutation relations. Nevertheless, the jump operators in Eq. (18) may be written similarly to Eq. (20) with the roles of $\cosh (r)$ and $\sinh (r)$ reversed. Because $|\cosh (r) / \sinh (r)|>1$, the jump operators contain a larger proportion of $\hat{a}_{\ell}^{\dagger}$ than $\hat{a}_{\ell}$, and the incoming two-qubit environment is more likely to transfer energy to the subsystems than to remove it. In this case the ME serves as an incoherent amplifier. In the following section we will investigate this parameter regime as well as the "exceptional points" where the bath is prepared in a maximally entangled state, e.g., a Bell state.

Note that when the bath qubits are prepared in the orthogonal subspace, $\left|\psi_{E}\right\rangle=\left|\psi_{E_{2}}\right\rangle\left(b_{\mathrm{gg}}=b_{\mathrm{ee}}=0\right)$, the cavity dynamics are qualitatively different from those analyzed above even when the bath qubits are near maximally entangled. In this case $\hat{L}_{1}=\hat{L}_{2}=0$ in Eq. (11), and the resulting ME has dissipative terms not related to squeezing.

In the following section we replace each optical cavity with a two-level atom coupled to its own environmental qubit (as opposed to interacting with a common environment such as the cases considered in [68-70]).

\section{TWO REMOTE TWO-LEVEL ATOMS INTERACTING WITH A BELL-STATE BATH}

In this section we investigate the repeated interaction between two remote two-level subsystems and a stream of maximally entangled bath qubits. The subsystems are taken to be identical, each described by a bare Hamiltonian $\hat{H}_{\ell}=$ $\frac{\omega_{0}}{2} \hat{\sigma}_{z, \ell}$, where $\omega_{0}$ is resonant with the bath qubit frequency, $\delta_{\ell}=\omega_{E_{\ell}}-\omega_{0}=0$. The interaction between each subsystem and its bath qubit is an excitation exchange described by a lowering operator, $\hat{c}_{\ell} \rightarrow \hat{\sigma}_{\ell}$. To avoid confusion, we henceforth refer to the bath as qubits and the subsystems as atoms, with the understanding that the bath qubits could indeed be physically manifested as a stream of entangled atoms.

\section{A. Bath qubits in a pure Bell state}

We first consider a maximally entangled two-qubit bath state in the subspace $\{|g g\rangle,|e e\rangle\}$,

$$
\left|\psi_{E}\right\rangle=\frac{1}{\sqrt{2}}\left(|\mathrm{ee}\rangle+e^{i \phi}|\mathrm{gg}\rangle\right),
$$

such that the Bell states $\left|\Phi_{E}^{+}\right\rangle$and $\left|\Phi_{E}^{-}\right\rangle$are given by $\phi=$ $(0, \pi)$, respectively. That is, the coefficients in Eq. (8) take values $b_{\mathrm{ee}}=1 / \sqrt{2}$ and $b_{\mathrm{gg}}=e^{i \phi} / \sqrt{2}$, while $b_{\mathrm{eg}}=b_{\mathrm{ge}}=0$. For simplicity we set $\lambda_{1}=\lambda_{2}=\lambda$ corresponding to decay rate $\gamma$, which yields the ME:

$$
\dot{\hat{\rho}}(t)=\frac{\gamma}{2}\left(\mathcal{D}\left[\hat{\sigma}_{1}+e^{i \phi} \hat{\sigma}_{2}^{\dagger}\right]+\mathcal{D}\left[e^{i \phi} \hat{\sigma}_{1}^{\dagger}+\hat{\sigma}_{2}\right] \hat{\rho}\right) .
$$


The joint state of the atomic subsystems is initialized in the arbitrary state

$$
\hat{\rho}_{0}=\sum_{j, k} \sum_{j^{\prime}, k^{\prime}} \rho_{j k, j^{\prime} k^{\prime}}^{0}|j k\rangle\left\langle j^{\prime} k^{\prime}\right|,
$$

where the sums run over $\{\mathrm{g}, \mathrm{e}\}$.

\section{Bath qubits in $\left|\Phi_{E}^{+}\right\rangle$}

First, we consider the case where the bath qubits are prepared in the Bell state, $\left|\Phi_{E}^{+}\right\rangle$, given by $\phi=0$ in Eq. (25). In the long-time limit, $t \rightarrow \infty$, the steady state of the two-atom system is given by

$$
\hat{\rho}_{\mathrm{ss}}=\left(\begin{array}{cccc}
\rho_{\mathrm{ee}, \mathrm{ee}}^{\mathrm{ss}} & 0 & 0 & \rho_{\mathrm{ee}, \mathrm{gg}}^{\mathrm{ss}} \\
0 & \rho_{\mathrm{eg}, \mathrm{eg}}^{\mathrm{ss}} & 0 & 0 \\
0 & 0 & \rho_{\mathrm{ge}, \mathrm{ge}}^{\mathrm{ss}} & 0 \\
\rho_{\mathrm{ee}, \mathrm{gg}}^{\mathrm{ss}} & 0 & 0 & \rho_{\mathrm{gg}, \mathrm{gg}}^{\mathrm{ss}}
\end{array}\right),
$$

where the steady-state matrix elements are related to the initial-state matrix elements by

$$
\begin{aligned}
\rho_{\mathrm{ee}, \mathrm{ee}}^{\mathrm{ss}} & =\frac{1}{3}\left[\rho_{\mathrm{ee}, \mathrm{ee}}^{0}+\rho_{\mathrm{gg}, \mathrm{gg}}^{0}-\rho_{\mathrm{ee}, \mathrm{gg}}^{0}+\frac{1}{2} \rho_{\mathrm{eg}, \mathrm{eg}}^{0}+\frac{1}{2} \rho_{\mathrm{ge}, \mathrm{ge}}^{0}\right] \\
\rho_{\mathrm{eg}, \mathrm{gg}}^{\mathrm{ss}} & =\frac{1}{3}\left[\frac{1}{2} \rho_{\mathrm{ee}, \mathrm{ee}}^{0}+\frac{1}{2} \rho_{\mathrm{gg}, \mathrm{gg}}^{0}+\rho_{\mathrm{ee}, \mathrm{gg}}^{0}+\rho_{\mathrm{eg}, \mathrm{eg}}^{0}+\rho_{\mathrm{ge}, \mathrm{ge}}^{0}\right], \\
\rho_{\mathrm{ge}, \mathrm{ge}}^{\mathrm{ss}} & =\rho_{\mathrm{eg}, \mathrm{eg}}^{\mathrm{ss}}, \\
\rho_{\mathrm{gg}, \mathrm{gg}}^{\mathrm{ss}} & =\rho_{\mathrm{ee}, \mathrm{ee}}^{\mathrm{ss}}, \\
\rho_{\mathrm{ee}, \mathrm{gg}}^{\mathrm{ss}} & =-\frac{1}{6}\left[\rho_{\mathrm{ee}, \mathrm{ee}}^{0}+\rho_{\mathrm{gg}, \mathrm{gg}}^{0}-4 \rho_{\mathrm{ee}, \mathrm{gg}}^{0}-\rho_{\mathrm{eg}, \mathrm{eg}}^{0}-\rho_{\mathrm{ge}, \mathrm{ge}}^{0}\right] .
\end{aligned}
$$

Thus the atomic steady state is not unique; rather, the ME has an invariant subspace. A particular steady state within this invariant subspace depends on the initial state [71-73].

The steady state of the joint atomic system can exhibit nonclassical correlations, identified by the negative partial transpose criterion [74]. The partial transpose matrix $\rho_{\mathrm{sS}}^{\mathrm{PT}}$, partitioned with respect to the subsystems, takes the following form:

$$
\hat{\rho}_{\mathrm{ss}}^{\mathrm{PT}}=\left(\begin{array}{cccc}
\rho_{\mathrm{ee}, \mathrm{ee}}^{\mathrm{ss}} & 0 & 0 & 0 \\
0 & \rho_{\mathrm{eg}, \mathrm{sg}}^{\mathrm{ss}} & \rho_{\mathrm{ee}, \mathrm{gg}}^{\mathrm{ss}} & 0 \\
0 & \rho_{\mathrm{gg}, \mathrm{ee}} & \rho_{\mathrm{ge}, \mathrm{ge}}^{\mathrm{ss}} & 0 \\
0 & 0 & 0 & \rho_{\mathrm{gg}, \mathrm{gg}}^{\mathrm{ss}}
\end{array}\right) .
$$

Negativity in the spectrum of $\hat{\rho}_{\mathrm{ss}}^{\mathrm{PT}}$ guarantees the presence of entanglement. We quantify the entanglement by the logarithmic negativity [75],

$$
L N(\hat{\rho}):=\log _{2}\left(\operatorname{Tr}\left[\sqrt{\hat{\rho}_{\mathrm{PT}}^{\dagger} \hat{\rho}_{\mathrm{PT}}}\right]\right),
$$

where $L N(\hat{\rho}) \in[0,1]$ with the minimum value corresponding to separable states and the maximum value to maximally entangled states.

A particular steady state of interest is when the atomic system is prepared in the Bell state $\left|\Phi^{-}\right\rangle$, whence it does not undergo any decoherence via interaction with the qubit bath, since it is already at the steady state. However, when the initial atomic state is $\left|\Phi^{+}\right\rangle$the steady state is the mixture

$$
\hat{\rho}_{\mathrm{ss}}=\frac{1}{3}\left(\left|\Phi^{+}\right\rangle\left\langle\Phi^{+}|+| \mathrm{eg}\right\rangle\langle\mathrm{eg}|+| \mathrm{ge}\rangle\langle\mathrm{ge}|\right),
$$

with a positive partial transpose matrix, i.e., it has purely positive eigenvalues $\left(\frac{1}{2}, \frac{1}{6}\right)$ with boldface indicating degeneracy (a)

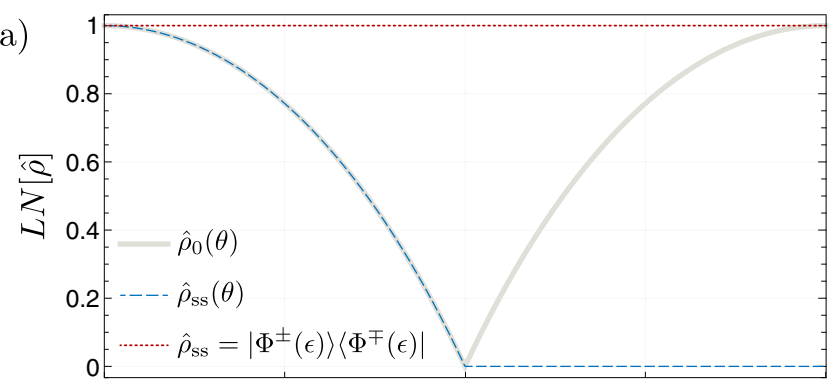

(b)

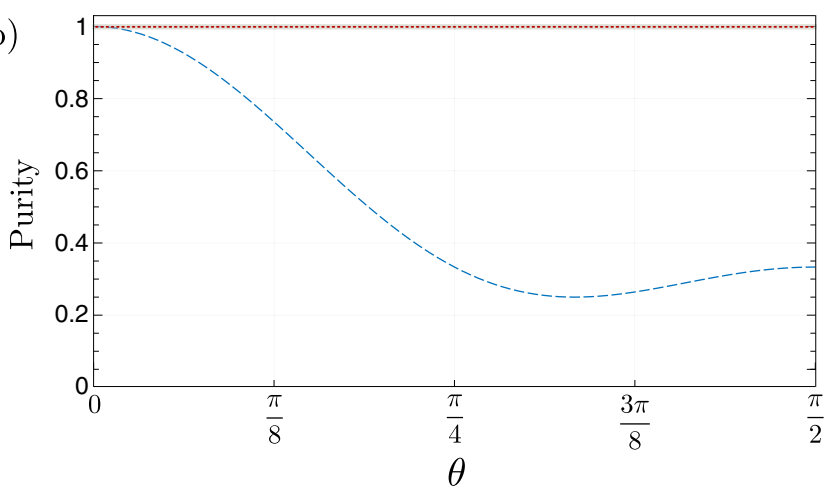

FIG. 3. Entanglement and purity of the atomic state when the two-qubit bath is prepared in the $\left|\Phi_{E}^{+}\right\rangle$Bell state. The initial twoatom state $\hat{\rho}_{0}(\theta)$ is parametrized by $\theta$, Eq. (32). We also include plots for nonmaximally entangled atomic states, $\left|\Phi^{ \pm}(\epsilon)\right\rangle$, that deviate from Bell states by $\epsilon=0.001$, Eq. (37). (a) Entanglement as quantified by the logarithmic negativity, Eq. (30), for which $L N(\hat{\rho}) \approx 1$. (b) State purity, $\operatorname{Tr}\left[\hat{\rho}^{2}\right]$. Note that, for $\theta<\theta_{c}=\pi / 4$, the gray curve and dashed blue curve exactly coincide. That is, initial entanglement is preserved despite the fact that the state becomes mixed. Beyond $\theta_{c}$ the steady state is no longer entangled and the minimum purity, 0.25 , occurs at $\theta=\pi / 3$.

of order 3. A positive partial transpose is a sufficient condition for separability of a two-qubit system.

An interesting scenario is an initial joint atomic state, $\hat{\rho}_{0}(\theta)=\left|\psi_{0}(\theta)\right\rangle\left\langle\psi_{0}(\theta)\right|$, that is a weighted sum of the two Bell states,

$$
\left|\psi_{0}(\theta)\right\rangle=\sin \theta\left|\Phi^{+}\right\rangle+\cos \theta\left|\Phi^{-}\right\rangle
$$

for $0 \leqslant \theta \leqslant \pi / 2$. Beyond this range in $\theta$, the entanglement behavior repeats. Above we found that the maximally entangled state, $\hat{\rho}_{0}(\theta=0)=\left|\Phi^{-}\right\rangle\left\langle\Phi^{-}\right|$, is a steady state of the ME. As $\theta$ deviates from zero, the contribution from the antisymmetric Bell state diminishes. Beyond the critical point $\theta_{c}=\pi / 4\left(\left|\psi_{0}\right\rangle=\mid\right.$ ee $\left.\rangle\right)$ the effect of the symmetric Bell state is dominant and the entanglement vanishes, $L N\left[\hat{\rho}_{\mathrm{ss}}(\theta \geqslant\right.$ $\left.\left.\theta_{c}\right)\right]=0$. At the critical point the steady state is a two-atom Werner state [76],

$$
\hat{\rho}_{\mathrm{ss}}\left(\theta_{c}\right)=\frac{1}{3}\left|\Phi^{-}\right\rangle\left\langle\Phi^{-}\right|+\frac{1}{6} \hat{\mathbb{1}}_{S},
$$

which is separable. In fact, even after this point the steady state is separable all the way to $\theta=\pi / 2$, at which point it is given by Eq. (31). The behavior is illustrated in Fig. 3(a) where we plot logarithmic negativity as a function of $\theta$ (dashed blue curve). Comparison with the initial logarithmic negativity (light gray curve) reveals that the Bell state environment 
TABLE I. Summary of results when the environment is prepared in a Bell state or a state very close to a Bell state, $\left|\Phi^{ \pm}(\epsilon)\right\rangle$, Eq. (37), or $\left|\Psi^{ \pm}(\epsilon)\right\rangle \equiv(\mid$ eg $\rangle \pm \sqrt{1+\epsilon} \mid$ ge $\left.\rangle\right) / \sqrt{2+\epsilon}$ parametrized by $0<\epsilon \ll 1$. The initial atomic state, Eq. (32), is parametrized by $\theta$ and numbers in bold indicate threefold degeneracy in the eigenvalue. When the two-qubit environment is exactly prepared in either of the two Bell states, the steady state $\hat{\rho}_{\mathrm{ss}}$ depends on the initial state $\hat{\rho}_{0}$ and the atoms remain entangled only for some specific parameter regimes. However, for an environment initialized in $\left|\Phi^{ \pm}(\epsilon)\right\rangle$ or $\left|\Psi^{ \pm}(\epsilon)\right\rangle$, the two atoms can acquire nonclassical correlations arbitrarily close to maximally entangled states.

\begin{tabular}{|c|c|c|c|c|}
\hline Two-qubit bath state $\hat{\rho}_{E}$ & $\theta$ & Initial atomic state $\hat{\rho}_{0}(\theta)$ & Steady state $\hat{\rho}_{\mathrm{ss}}(\theta)$ & Spectrum of $\hat{\rho}_{\mathrm{ss}}^{\mathrm{PT}}(\theta)$ \\
\hline$\left|\Phi^{+}\right\rangle\left\langle\Phi^{+}\right|$ & 0 & $\left|\Phi^{-}\right\rangle\left\langle\Phi^{-}\right|$ & $\left|\Phi^{-}\right\rangle\left\langle\Phi^{-}\right|$ & $\frac{1}{2}(-1, \mathbf{1})$ \\
\hline$"$ & $\theta_{c}=\frac{\pi}{4}$ & $|e e\rangle\langle e e|$ & $\frac{1}{6} \hat{\mathbb{1}}_{S}+\frac{1}{3}\left|\Phi^{-}\right\rangle\left\langle\Phi^{-}\right|$ & $\left(\frac{1}{2}, \frac{1}{6}\right)$ \\
\hline$"$ & $\frac{\pi}{2}$ & $\left|\Phi^{+}\right\rangle\left\langle\Phi^{+}\right|$ & $\frac{1}{3}\left(|\mathrm{eg}\rangle\langle\mathrm{eg}|+| \mathrm{ge}\rangle\left\langle\mathrm{ge}|+| \Phi^{+}\right\rangle\left\langle\Phi^{+}\right|\right)$ & $\frac{1}{3}(0, \mathbf{1})$ \\
\hline$\left|\Phi^{-}\right\rangle\left\langle\Phi^{-}\right|$ & 0 & $\left|\Phi^{-}\right\rangle\left\langle\Phi^{-}\right|$ & $\frac{1}{3}\left(|\mathrm{eg}\rangle\langle\mathrm{eg}|+| \mathrm{ge}\rangle\left\langle\mathrm{ge}|+| \Phi^{-}\right\rangle\left\langle\Phi^{-}\right|\right)$ & $\frac{1}{3}(0, \mathbf{1})$ \\
\hline$"$ & $\theta_{c}$ & $|e e\rangle\langle e e|$ & $\frac{1}{6} \hat{\mathbb{1}}_{S}+\frac{1}{3}\left|\Phi^{+}\right\rangle\left\langle\Phi^{+}\right|$ & $\left(\frac{1}{2}, \frac{1}{6}\right)$ \\
\hline 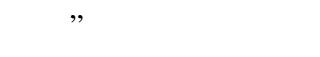 & $\frac{\pi}{2}$ & $\left|\Phi^{+}\right\rangle\left\langle\Phi^{+}\right|$ & $\left|\Phi^{+}\right\rangle\left\langle\Phi^{+}\right|$ & $\frac{1}{2}(-1, \mathbf{1})$ \\
\hline$\left|\Phi^{ \pm}(\epsilon)\right\rangle\left\langle\Phi^{ \pm}(\epsilon)\right|$ & $\in\left[0, \frac{\pi}{2}\right]$ & $\left|\psi_{0}(\theta)\right\rangle\left\langle\psi_{0}(\theta)\right|$ & $\left|\Phi^{\mp}(\epsilon)\right\rangle\left\langle\Phi^{\mp}(\epsilon)\right|$ & $\left(\frac{1}{2+\epsilon}, \frac{1+\epsilon}{2+\epsilon}, \frac{1}{2},-\frac{1}{2}\right)$ \\
\hline$\left|\Psi^{+}\right\rangle\left\langle\Psi^{+}\right|$ & 0 & $\left|\Psi^{-}\right\rangle\left\langle\Psi^{-}\right|$ & $\left|\Psi^{-}\right\rangle\left\langle\Psi^{-}\right|$ & $\frac{1}{2}(-1, \mathbf{1})$ \\
\hline 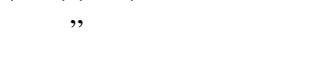 & $\theta_{c}$ & $|\mathrm{eg}\rangle\langle\mathrm{eg}|$ & $\frac{1}{6} \hat{\mathbb{1}}_{S}+\frac{1}{3}\left|\Psi^{-}\right\rangle\left\langle\Psi^{-}\right|$ & $\left(\frac{1}{2}, \frac{1}{6}\right)$ \\
\hline$"$ & $\frac{\pi}{2}$ & $\left|\Psi^{+}\right\rangle\left\langle\Psi^{+}\right|$ & $\frac{1}{3}(\mid$ ee $\rangle\langle$ ee $\left.|+| \mathrm{gg}\rangle\left\langle\mathrm{gg}|+| \Psi^{+}\right\rangle\left\langle\Psi^{+}\right|\right)$ & $\frac{1}{3}(0, \mathbf{1})$ \\
\hline$\left|\Psi^{-}\right\rangle\left\langle\Psi^{-}\right|$ & 0 & $\left|\Psi^{-}\right\rangle\left\langle\Psi^{-}\right|$ & $\frac{1}{3}(\mid$ ee $\rangle\langle$ ee $\left.|+| \mathrm{gg}\rangle\left\langle\mathrm{gg}|+| \Psi^{-}\right\rangle\left\langle\Psi^{-}\right|\right)$ & $\frac{1}{3}(0, \mathbf{1})$ \\
\hline$"$ & $\theta_{c}$ & $|\mathrm{eg}\rangle\langle\mathrm{eg}|$ & $\frac{1}{6} \hat{\mathbb{1}}_{S}+\frac{1}{3}\left|\Psi^{+}\right\rangle\left\langle\Psi^{+}\right|$ & $\left(\frac{1}{2}, \frac{1}{6}\right)$ \\
\hline ” & $\frac{\pi}{2}$ & $\left|\Psi^{+}\right\rangle\left\langle\Psi^{+}\right|$ & $\left|\Psi^{+}\right\rangle\left\langle\Psi^{+}\right|$ & $\frac{1}{2}(-1, \mathbf{1})$ \\
\hline$\left|\Psi^{ \pm}(\epsilon)\right\rangle\left\langle\Psi^{ \pm}(\epsilon)\right|$ & $\in\left[0, \frac{\pi}{2}\right]$ & $\left|\psi_{0}(\theta)\right\rangle\left\langle\psi_{0}(\theta)\right|$ & $\left|\Psi^{\mp}(\epsilon)\right\rangle\left\langle\Psi^{\mp}(\epsilon)\right|$ & $\left(\frac{1}{2+\epsilon}, \frac{1+\epsilon}{2+\epsilon}, \frac{1}{2},-\frac{1}{2}\right)$ \\
\hline
\end{tabular}

preserves the initial system entanglement up until $\theta_{c}$ despite the fact that the state becomes mixed; see Fig. 3(b).

\section{Bath qubits in $\left|\Phi_{E}^{-}\right\rangle,\left|\Psi_{E}^{+}\right\rangle$, or $\left|\Psi_{E}^{-}\right\rangle$}

The situation where the bath is prepared in $\left|\Phi_{E}^{-}\right\rangle$proceeds similarly. In this case, the steady state has the same form as Eq. (28) with the following substitutions:

$$
\rho_{\mathrm{ee}, \mathrm{gg}}^{0} \rightarrow-\rho_{\mathrm{ee}, \mathrm{gg}}^{0} \quad \text { and } \quad \rho_{\mathrm{ee}, \mathrm{gg}}^{\mathrm{ss}} \rightarrow-\rho_{\mathrm{ee}, \mathrm{sg}}^{\mathrm{ss}} .
$$

When the initial atomic state is parametrized as in Eq. (32), the steady states for the extremal cases, $\theta=\left(0, \theta_{c}\right)$, are just as in Eq. (31) and Eq. (33), respectively, with the roles of $\left|\Phi^{ \pm}\right\rangle$ swapped. By varying $\theta$ in the interval $\left[0, \theta_{c}\right]$, the antisymmetric Bell state is dominant, the result of which is that the systems remain separable. For the range $\theta>\theta_{c}$ the symmetric Bell state, $\left|\Phi^{+}\right\rangle$, is dominant in the initial atomic state and the ME preserves the initial entanglement. For $\theta=\pi / 2$ the initial state, $\rho_{0}(\theta=\pi / 2)=\left|\Phi^{+}\right\rangle\left\langle\Phi^{+}\right|$, is a steady state.

Lastly, when the qubit environment is prepared in the other subspace $\{\mid$ eg $\rangle$, |ge $\rangle$,

$$
\left|\psi_{E}\right\rangle=\frac{1}{\sqrt{2}}\left(|\mathrm{eg}\rangle+e^{i \phi}|\mathrm{ge}\rangle\right),
$$

with Bell states $\left|\Psi_{E}^{+}\right\rangle$and $\left|\Psi_{E}^{-}\right\rangle$given by $\phi=\{0, \pi\}$, the steady states have an analogous form. Table I summarizes the results for comparison.

\section{B. Bath qubits in a nonmaximally entangled state}

From the above analysis it may be inferred that the existence of coherences in the environment is merely a necessary condition for entangling the subsystems, even though the cross terms in the master equation, Eq. (26), might suggest otherwise, i.e., the generation of quantum correlation among systems. We found that Bell-state baths generate a ME without a unique steady state and, depending on the initial atomic state, atomic entanglement is either preserved or destroyed, but never created. Here we show that, when the bath qubits are prepared in a nonmaximally entangled state that can be arbitrarily close to a Bell state, the ME has a unique, entangled steady state. Consider the bath in the following state that slightly deviates from a maximally entangled state:

$$
\left|\psi_{E}(\phi, \epsilon)\right\rangle=\frac{1}{\sqrt{2+\epsilon}}\left(|\mathrm{ee}\rangle+e^{i \phi} \sqrt{1+\epsilon}|\mathrm{gg}\rangle\right) .
$$

The atomic steady state is highly entangled for all $\phi$, with logarithmic negativity, $L N(\hat{\rho}) \approx 1$ for $0<\epsilon \ll 1$. This can be seen by considering the following approximate Bell states for the qubit bath:

$$
\begin{aligned}
\left|\Phi^{+}(\epsilon)\right\rangle & :=\left|\psi_{E}(0, \epsilon)\right\rangle, \\
\left|\Phi^{-}(\epsilon)\right\rangle & :=\left|\psi_{E}(\pi, \epsilon)\right\rangle .
\end{aligned}
$$

The corresponding atomic steady states are the pure states, $\hat{\rho}_{\mathrm{ss}}=\left|\Phi^{\mp}(\epsilon)\right\rangle\left\langle\Phi^{\mp}(\epsilon)\right|$. The partial transpose matrices $\hat{\rho}_{\mathrm{ss}}^{\mathrm{PT}}$ have respective eigenvalues

$$
\left(\frac{1}{2+\epsilon}, \frac{1+\epsilon}{2+\epsilon}, \frac{1}{2},-\frac{1}{2}\right)
$$

that guarantee entanglement. The logarithmic negativity of the steady states is shown in Fig. 3 for $\epsilon=0.001$ (dotted red line).

This behavior continues for the bath qubits far from Bell states. In Fig. 4 we plot the negativity of the atomic steady 


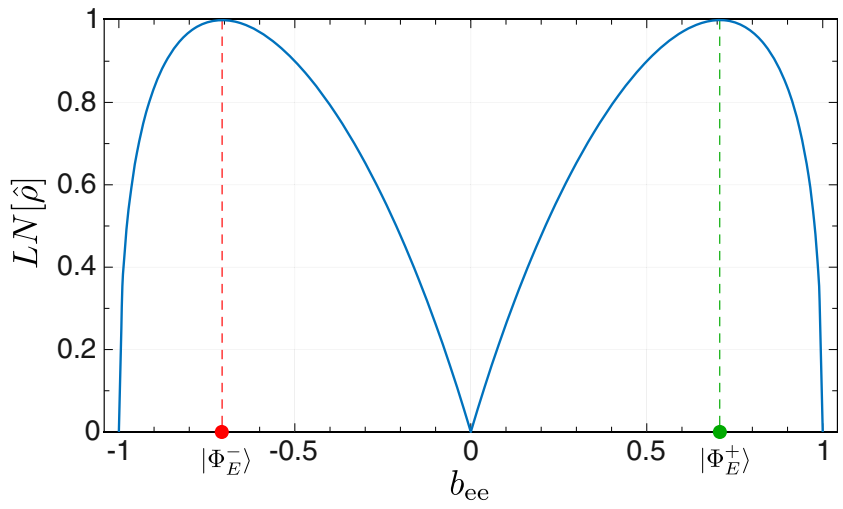

FIG. 4. Steady-state entanglement of two remote atoms interacting with a two-qubit bath, quantified by the logarithmic negativity $L N\left(\hat{\rho}_{\mathrm{ss}}\right)$. The qubit bath is parametrized by Eq. (8a) with $b_{\text {ee }}$ and $b_{\mathrm{gg}}$ taken to be real. At the exceptional points, where the bath is prepared in a Bell state, $b_{\text {ee }}= \pm 1 / \sqrt{2}$ indicated by red and green dots, the atomic steady state is not unique. In this case, the steadystate entanglement is a function of the initial atomic state (see Fig. 3).

state for the two-qubit bath state given by Eq. (8a), with $b_{\mathrm{ee}}$ and $b_{\mathrm{gg}}$ taken to be real.

For $b_{\mathrm{ee}}=\{0, \pm 1\}$ the qubit bath state is separable, and as expected, the atoms relax to an uncorrelated steady state. As the entanglement in the bath qubits increases, so does that of the atomic steady state. The logarithmic negativity approaches a limiting value of $L N\left(\hat{\rho}_{\mathrm{ss}}\right) \rightarrow 1$ as the bath approaches a Bell state, $b_{\mathrm{ee}}=\lim _{\epsilon \rightarrow 0} \pm 1 / \sqrt{2+\epsilon}$. Indeed, for each value of $b_{\mathrm{ee}}$, the atomic steady state has the same logarithmic negativity as is present in the two-qubit bath state, indicated by full distribution of environmental entanglement to the atoms. As discussed in Sec. V A at $b_{\mathrm{ee}}= \pm 1 / \sqrt{2}$ (red and green dots), where the environment is exactly in the Bell states, the steadystate atoms can exhibit any value of the logarithmic negativity, depending on their initial state. Recall that a similar situation arose for the case of two remote cavities in Sec. IV. We found that when the bath qubits were prepared in the $\left|\Phi_{E}^{ \pm}\right\rangle$Bell state, this represented the limit of infinite squeezing for the cavities' steady state.

\section{GENERAL CASE: MASTER EQUATIONS FOR $n$-QUBIT ENVIRONMENTS}

Our focus thus far has been on the system dynamics and steady-state properties generated by two-qubit environments. A natural question is how does the evolution differ when the environment consists of more than two qubits? In order to address this, we expand the formalism presented in Sec. III to the case of $n$-qubit environments. We present the ME for $n$ qubit environments and illustrate some key differences when going beyond two-qubit baths.

Let us now proceed by supposing that all approximations required for the ME derivation in Sec. III (Born Markov, weak coupling, and $\left.\operatorname{Tr}\left[\hat{\sigma}_{\ell} \hat{\rho}_{E}\right]=0\right)$ are applicable here as well. At each interaction time interval the environment is prepared in a general $n$-qubit state $\hat{\rho}_{E}$. It is straightforward to generalize the master equation derived for the two-qubit environments, Eq. (13), to the $n$-qubit scenario. Following the

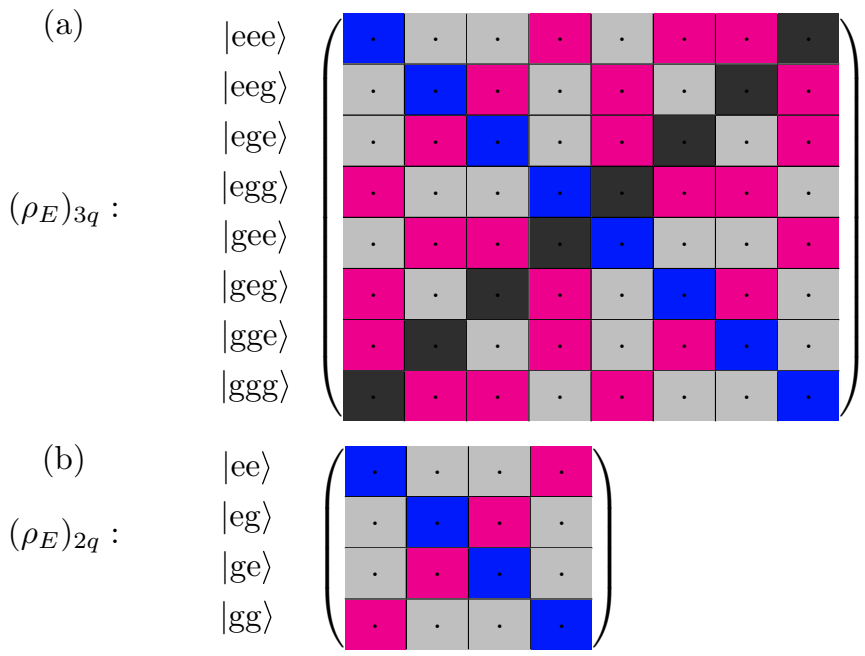

FIG. 5. Color-coded representation of the state matrix for threeand two-qubit environments. Matrix elements in the local energy basis contribute to different terms in the multiqubit ME, Eqs. (39) and (40). Matrix elements labeled in gray (*) are zero because of the form of the environment's state, Eq. (8). Those labeled in dark blue $(\square)$ contribute to local dissipators, $\mathcal{D}[\hat{o}] \hat{\rho}$, and those labeled in magenta (-) contribute to the two-body superoperators, $\mathcal{S}\left[\hat{o}_{\ell}, \hat{o}_{m}\right] \hat{\rho}$. Matrix elements labeled in black (-) do not contribute to the ME dynamics. Note the similarities to Fig. 3 in Ref. [29].

same procedure Eq. (5a) is substituted into Eq. (6) so that the evolution of the joint $n$ system is described by the following ME:

$$
\begin{aligned}
\dot{\hat{\rho}}(t)= & \sum_{\ell=1}^{n}\left(\gamma_{\downarrow, \ell} \mathcal{D}\left[\hat{c}_{\ell}\right]+\gamma_{\uparrow, \ell} \mathcal{D}\left[c_{\ell}^{\dagger}\right]\right) \hat{\rho} \\
& +\sum_{\ell<m}\left(\gamma_{\downarrow \downarrow, \ell m} \mathcal{S}\left[\hat{c}_{\ell}, \hat{c}_{m}\right]+\gamma_{\downarrow \uparrow, \ell m} \mathcal{S}\left[\hat{c}_{\ell}, \hat{c}_{m}^{\dagger}\right]\right. \\
& \left.+\gamma_{\downarrow \uparrow, \ell m}^{*} \mathcal{S}\left[\hat{c}_{\ell}^{\dagger}, \hat{c}_{m}\right]+\gamma_{\downarrow \downarrow, \ell m}^{*} \mathcal{S}\left[\hat{c}_{\ell}^{\dagger}, \hat{c}_{m}^{\dagger}\right]\right) \hat{\rho},
\end{aligned}
$$

where

$$
\begin{aligned}
\gamma_{\downarrow, \ell} & =\gamma_{\ell} \operatorname{Tr}_{E}\left[\left\langle\mathrm{~g}_{\ell}\left|\hat{\rho}_{E}\right| \mathrm{g}_{\ell}\right\rangle\right], \\
\gamma_{\uparrow, \ell} & =\gamma_{\ell} \operatorname{Tr}_{E}\left[\left\langle\mathrm{e}_{\ell}\left|\hat{\rho}_{E}\right| \mathrm{e}_{\ell}\right\rangle\right], \\
\gamma_{\downarrow \downarrow, \ell m} & =\sqrt{\gamma_{\ell} \gamma_{m}} \operatorname{Tr}_{E}\left[\left\langle\mathrm{~g}_{\ell}, \mathrm{g}_{m}\left|\hat{\rho}_{E}\right| \mathrm{e}_{\ell}, \mathrm{e}_{m}\right\rangle\right], \\
\gamma_{\downarrow \uparrow, \ell m} & =\sqrt{\gamma_{\ell} \gamma_{m}} \operatorname{Tr}_{E}\left[\left\langle\mathrm{~g}_{\ell}, \mathrm{e}_{m}\left|\hat{\rho}_{E}\right| \mathrm{e}_{\ell}, \mathrm{g}_{m}\right\rangle\right],
\end{aligned}
$$

where for example $\left|\mathrm{e}_{\ell}, \mathrm{e}_{m}\right\rangle$ is the excited bath state for subsystems $\ell$ and $m$. The above ME can also be straightforwardly expressed in diagonal, Lindblad form with $2 n(n-1)$ jump operators; however, the form above is more amenable when considering mixed-state baths.

The ME in Eq. (39) has two physical mechanisms (which are independent of $n$ ): (1) processes involving the gain $(\uparrow)$ or loss $(\downarrow)$ of a system excitation via local dissipators and (2) two-excitation processes described by superoperators $\mathcal{S}\left[\hat{o}_{\ell}, \hat{o}_{m}\right] \hat{\rho}$. When the $n$-qubit bath state is expressed in the local energy basis, each matrix element contributes to one of these two processes through the coefficients in Eq. (40) or does not drive dynamics at all. Indicated in Fig. 5 are the 
contribution of the environmental state matrix elements to each of these processes (in dark blue and magenta, respectively), for two- and three-qubit environments. The entries of $\hat{\rho}_{E}$ shown by gray color are zero due to the assumption that the expectation value of the bath operators over $\hat{\rho}_{E}$ should be zero.

Consider first the $n=3$ bath. An important observation is the absence of antidiagonal matrix elements, indicated by black cells in Fig. 5(a), in any coefficients in Eq. (40). Surprisingly, this implies that preparing the environmental qubits in certain maximally entangled states such as the Greenberger-Horne-Zeilinger (GHZ) state [77] in the local energy basis is not useful for generating correlations between the subsystems for $n>2$. Additionally, if the quantum systems are atoms prepared in a GHZ state, their nonclassical quantum correlations eventually decohere, as expected for three subsystems subject to local dissipation such as dephasing or depolarizing channels [44]. This is in marked contrast to two-qubit baths where the antidiagonal components of $\hat{\rho}_{E}$ that appear in Eqs. (15e) and (15f), indicated by the magenta cells in Fig. 5(b), are the key ingredient for entangling the systems. This highlights the significance of the two-qubit $X$-state environments (described by a state matrix in which only diagonal and antidiagonal entries are nonzero) $[44,45]$. Dağ et al. [29] encountered a similar result-they found that antidiagonal coherences in $n=3$ qubit baths do not contribute to squeezing or displacement of a single cavity mode.

This behavior extends to $n$-qubit baths. The antidiagonal matrix elements of $\hat{\rho}_{E}$ have the form $|x\rangle\langle\bar{x}|$ where $x$ is a string of $\mathrm{e}$ and $\mathrm{g}$ labels and $\bar{x}$ is the complement string with e and $\mathrm{g}$ swapped. For $n \geqslant 3$ these matrix elements do not contribute the coefficients in Eq. (40)-either the inner product is zero or the trace over the remaining systems is zero. Thus, for $n \geqslant 3$, the qubit correlations in an $X$-state bath play no part in the ME dynamics and cannot influence entanglement among the subsystems. In fact, the influence of any bath-qubit correlations beyond two-body entanglement vanishes in the ME. As a result, many highly entangled bath states, including maximally entangled states, do not play a role in entangling the subsystems.

This analysis suggests a way that could be used for steady-state entanglement across all subsystems, which could be verified by calculating an $n$-partite entanglement witness [78-82]. Since the ME, given in Eq. (39), has only two-body cross terms, generating entanglement among the subsystems might be achieved by engineering a specific structure in $\hat{\rho}_{E}$. It seems the presence of pairwise entanglement in a particular form in the bath qubits is sufficient to make sure that the coefficients in Eqs. (40c) and (40d) are nonzero. It remains an open question whether such an environment with pairwise entanglement would enable entangling dynamics in the ME, or perhaps more generally a sequence of entangled environments operating for consecutive periods of time. In addition, the cost of engineering such an environment would be an important factor. Another interesting case is three-qubit entangled $W$ state baths which retain some two-body superoperators in the ME. However, to determine whether the subsystems become entangled in the long-time limit, a thorough analysis like the one presented in Sec. V would be required. There, PPT is just a necessary condition, and calculating logarithmic negativity for three bipartitions of the system is required.

Although the absence of coupling from antidiagonal coherences in $\hat{\rho}_{E}$ may appear puzzling, some of the observed structure will be due to the weak-coupling approximation that underpins the master equation derivation. Recall that the joint state of the systems is updated by a dynamical map, Eq. (6), that results from tracing out the bath after unitary evolution over a small interaction time $\Delta t$. In the weak-coupling regime the expansion of the unitary operator, Eq. (5a), is truncated at the second-order term in $\Delta t$. This limits the influence of bath correlations in the ME to two-body terms. If one were to keep terms up to $\Delta t^{3}$ or beyond, these coherences would play a part in the dynamical map. This could arise when the coupling time between each successive qubit and its corresponding subsystem is large enough that weak-coupling criterion, $\lambda_{i} \Delta t \ll 1$, is not entirely valid and requires perturbative corrections.

\section{CONCLUSION}

Within the repeated quantum interaction formalism we have derived master equations for open quantum systems evolving under irreversible entangled quantum channels. The environment is composed of a chain of identical entangled two-level systems which sequentially interact weakly with their corresponding subsystems and are then discarded. In the limit of a continuous stream of environmental qubits, the joint system evolves according to a Markovian ME with local effective Hamiltonians and nonlocal dissipative processes with jump operators that are combinations of creation and destruction operators across the subsystems. This description applies generally to a qubit bath in a mixed state, for which we provide an alternate, nondiagonal form of the ME that can be easier to work with.

A pedagogical study of a two-qubit bath coupled to a pair of two-level subsystems led to several conclusions. First, we find that the presence of antidiagonal coherences in the bath is essential for steady-state entanglement of the subsystems, when the bath state is expressed in a basis of local eigenstates. Second, maximally entangled bath states do not give rise to unique steady states while even slight deviations from these "exceptional" bath states do.

For the general case of entangled $n$-qubit baths, the ME contains at most two-body terms in the jump operators. A surprising consequence is that particular maximally entangled $n$ qubit baths (for $n>2$ ) do not affect subsystem entanglement. That is, when expressed in the local energy basis antidiagonal coherences in the bath state do not couple to the system. An implication is that $X$-state baths drive the same dynamics as diagonal state baths such as a thermal bath. This is in contrast to the two-qubit environments where the existence of antidiagonal coherences are essential to the generation of entanglement between the systems.

This work opens several avenues for future research. Extending the methods of Gross et al. [32], the formalism presented here offers a way to model multimode Gaussian bosonic baths such as two-mode squeezed electromagnetic environments. Thermal and other mixed-state baths can be directly modeled by tracing over a part of a multiqubit entangled bath. Moreover, it is possible to proceed beyond 
Gaussian baths by perturbatively extending the weak-coupling limit with the inclusion of higher-order terms, $O\left[(\lambda \Delta t)^{k}\right]$, in the interaction Hamiltonian. Detuning the bath qubits from the systems, $\delta_{\ell} \neq 0$, may also generate interesting dynamics [70,83].

Environment-assisted entangling protocols based on engineered qubit environments that include only pairwise entanglement across all qubits could be used to create useful multipartite entanglement among all systems (generating cluster states, for example). This might be useful especially when entangling the multipartite system in other ways is practically challenging. Several extensions to the bath itself could be studied. The bath qubits could be replaced by $d$-dimensional quantum systems, or qudits, yielding a richer structure to the environment, and simultaneous spatial entanglement between qubits across channels and entanglement in time between progressive qubits in a single channel such that the evolution is inherently nonlocal and non-Markovian [28,38,49].

\section{ACKNOWLEDGMENTS}

B.Q.B. thanks Nicolas Menicucci and Rafael Alexander for valuable discussions. This project was supported in part by the Australian Research Council (ARC) Centre of Excellence for Engineered Quantum Systems (Grants No. CE1 10001013 and No. CE170100009). B.Q.B. also acknowledges support from the ARC Centre of Excellence for Quantum Computation and Communication Technology (Project No. CE170100012).

\section{APPENDIX A: MASTER EQUATION DERIVATION FOR A TWO-QUBIT ENVIRONMENT}

Inserting the unitary interaction, $\hat{U}_{I}^{(k)}$, from Eq. (5a) into the dynamical map in Eq. (6) and keeping terms to second order in $\Delta t$, the following expression is obtained [28,29,31]:

$$
\hat{\rho}\left(t_{k+1}\right)=\operatorname{Tr}_{E}\left(\hat{\rho}\left(t_{k}\right) \otimes \hat{\rho}_{E}-i \Delta t\left[\hat{H}_{I}^{(k)}, \hat{\rho}\left(t_{k}\right) \otimes \hat{\rho}_{E}\right]+\Delta t^{2} \hat{H}_{I}^{(k)} \hat{\rho}\left(t_{k}\right) \otimes \hat{\rho}_{E} \hat{H}_{I}^{(k)}-\frac{\Delta t^{2}}{2}\left\{\left(\hat{H}_{I}^{(k)}\right)^{2}, \hat{\rho}\left(t_{k}\right) \otimes \hat{\rho}_{E}\right\}_{+}\right)
$$

where $\{\hat{A}, \hat{B}\}_{+}$denotes an anticommutator. We will explicitly take the environmental trace for each term in the expansion with respect to the environment, $\hat{\rho}_{E}=\sum_{l}\left|\psi_{E_{l}}\right\rangle\left\langle\psi_{E_{l}}\right|$, where $\left|\psi_{E_{l}}\right\rangle$ are given in Eq. (8). The first term is simply just the system state $\hat{\rho}\left(t_{k}\right)$. The commutator term proportional to $\Delta t$ vanishes because we assume $\operatorname{Tr}\left[\hat{\sigma}_{\ell} \hat{\rho}_{E}\right] \equiv\left\langle\hat{\sigma}_{\ell}\right\rangle=0$,

$$
\operatorname{Tr}_{E}\left(\left[\hat{H}_{I}^{(k)}, \hat{\rho}\left(t_{l}\right) \otimes \hat{\rho}_{E}\right]\right)=\left[\lambda_{1} \hat{c}_{1}\left\langle\hat{\sigma}_{1}^{\dagger}\right\rangle+\lambda_{2} \hat{c}_{2}\left\langle\hat{\sigma}_{2}^{\dagger}\right\rangle+\text { H.c., } \hat{\rho}\left(t_{k}\right)\right]=0 .
$$

Now we turn our attention to the second-order terms, proportional to $\Delta t^{2}$. The first term in the second line becomes

$$
\begin{aligned}
\operatorname{Tr}_{E}\left(\hat{H}_{I}^{(k)} \hat{\rho}\left(t_{k}\right) \otimes \hat{\rho}_{E} \hat{H}_{I}^{(k)}\right)= & \lambda_{1}^{2}\left\{\left(\left|b_{\mathrm{ge}}\right|^{2}+\left|b_{\mathrm{gg}}\right|^{2}\right) \hat{c}_{1} \hat{\rho}\left(t_{k}\right) \hat{c}_{1}^{\dagger}+\left(\left|b_{\mathrm{ee}}\right|^{2}+\left|b_{\mathrm{eg}}\right|^{2}\right) \hat{c}_{1}^{\dagger} \hat{\rho}\left(t_{k}\right) \hat{c}_{1}\right\} \\
& +\lambda_{2}^{2}\left\{\left(\left|b_{\mathrm{eg}}\right|^{2}+\left|b_{\mathrm{gg}}\right|^{2}\right) \hat{c}_{2} \hat{\rho}\left(t_{k}\right) \hat{c}_{2}^{\dagger}+\left(\left|b_{\mathrm{ee}}\right|^{2}+\left|b_{\mathrm{ge}}\right|^{2}\right) \hat{c}_{2}^{\dagger} \hat{\rho}\left(t_{k}\right) \hat{c}_{2}\right\} \\
& +\lambda_{1} \lambda_{2}\left\{b_{\mathrm{gg}} b_{\mathrm{ee}}^{*} \hat{c}_{1} \hat{\rho}\left(t_{k}\right) \hat{c}_{2}+b_{\mathrm{ge}} b_{\mathrm{eg}}^{*} \hat{c}_{1} \hat{\rho}\left(t_{k}\right) \hat{c}_{2}^{\dagger}+b_{\mathrm{ee}} b_{\mathrm{gg}}^{*} \hat{c}_{1}^{\dagger} \hat{\rho}\left(t_{k}\right) \hat{c}_{2}^{\dagger}+b_{\mathrm{eg}} b_{\mathrm{ge}}^{*} \hat{c}_{1}^{\dagger} \hat{\rho}\left(t_{k}\right) \hat{c}_{2}+\mathrm{H} . \mathrm{c} .\right\}
\end{aligned}
$$

And the remaining anticommutator term becomes

$$
\begin{aligned}
\operatorname{Tr}_{E}\left[\left\{\left(\hat{H}_{I}^{(k)}\right)^{2}, \hat{\rho}\left(t_{k}\right) \otimes \hat{\rho}_{E}\right\}_{+}\right]= & \lambda_{1}^{2}\left\{\left(\left|b_{\mathrm{ee}}\right|^{2}+\left|b_{\mathrm{eg}}\right|^{2}\right)\left\{\hat{\rho}\left(t_{k}\right), \hat{c}_{1} \hat{c}_{1}^{\dagger}\right\}_{+}+\left(\left|b_{\mathrm{ge}}\right|^{2}+\left|b_{\mathrm{gg}}\right|^{2}\right)\left\{\hat{\rho}\left(t_{k}\right), \hat{c}_{1}^{\dagger} \hat{c}_{1}\right\}_{+}\right\} \\
& +\lambda_{2}^{2}\left\{\left(\left|b_{\mathrm{ee}}\right|^{2}+\left|b_{\mathrm{ge}}\right|^{2}\right)\left\{\hat{\rho}\left(t_{k}\right), \hat{c}_{2} \hat{c}_{2}^{\dagger}\right\}_{+}+\left(\left|b_{\mathrm{eg}}\right|^{2}+\left|b_{\mathrm{gg}}\right|^{2}\right)\left\{\hat{\rho}\left(t_{k}\right), \hat{c}_{2}^{\dagger} \hat{c}_{2}\right\}_{+}\right\} \\
& +2 \lambda_{1} \lambda_{2}\left\{b_{\mathrm{gg}} b_{\mathrm{ee}}^{*}\left\{\hat{\rho}\left(t_{k}\right), \hat{c}_{1} \hat{c}_{2}\right\}_{+}+b_{\mathrm{ge}} b_{\mathrm{eg}}^{*}\left\{\hat{\rho}\left(t_{k}\right), \hat{c}_{1} \hat{c}_{2}^{\dagger}\right\}_{+}\right. \\
& \left.+b_{\mathrm{ee}} b_{\mathrm{gg}}^{*}\left\{\hat{\rho}\left(t_{k}\right), \hat{c}_{1}^{\dagger} \hat{c}_{2}^{\dagger}\right\}_{+}+b_{\mathrm{eg}} b_{\mathrm{ge}}^{*}\left\{\hat{\rho}\left(t_{k}\right), \hat{c}_{1}^{\dagger} \hat{c}_{2}\right\}_{+}\right\} .
\end{aligned}
$$

Now putting all terms together we can rewrite Eq. (A1) as

$$
\hat{\rho}\left(t_{k+1}\right)=\left(\mathcal{I}+\Delta t^{2} \mathcal{E}\right) \hat{\rho}\left(t_{k}\right) \equiv \mathcal{M}(\Delta t) \hat{\rho}\left(t_{k}\right),
$$

where $\mathcal{I}$ is the identity map and $\mathcal{E}=\mathcal{E}\left(\lambda_{1}, \lambda_{2}\right)$ is a linear operator on $\hat{\rho}$. The map $\mathcal{M}$ is divisible with

$$
\begin{aligned}
\hat{\rho}\left(t_{k+1}\right) & =\left(\mathcal{I}+\frac{1}{n} \Delta t^{2} \mathcal{E}\right)^{n} \hat{\rho}\left(t_{k}\right) \equiv\left[\mathcal{M}_{n}(\Delta t)\right]^{n} \hat{\rho}\left(t_{k}\right) \\
& =\mathcal{M}(\Delta t) \hat{\rho}\left(t_{k}\right)+O\left(\Delta t^{4}\right), \quad \forall n
\end{aligned}
$$

So we cannot distinguish between the original map and $n$ applications of the $\frac{1}{n}$-strength map. We emphasize that scaling down the time step $\Delta t$ by $n$ and applying the map $n$ times does not reproduce the same dynamics as does $n$ applications of $\left[\mathcal{M}_{n}(\Delta t)\right]$, as it is clear from the above equations. That is,

$$
[\mathcal{M}(\Delta t / n)]^{n} \hat{\rho}\left(t_{k}\right) \neq\left[\mathcal{M}_{n}(\Delta t)\right]^{n} \hat{\rho}\left(t_{k}\right) .
$$

Let us now update the system's state for one time increment $\tau=\Delta t / n$ (which can be arbitrarily small) so we have

$$
\hat{\rho}(t+\tau)=(\mathcal{I}+\tau \Delta t \mathcal{E}) \hat{\rho}(t)
$$

where we assume $t_{k}=t$. It is then easy to construct a time derivative of the state according to

$$
\lim _{\tau \rightarrow 0} \frac{\hat{\rho}(t+\tau)-\hat{\rho}(t)}{\tau}=\dot{\hat{\rho}}(t)=\Delta t \mathcal{E} \hat{\rho}(t) .
$$


The right-hand side is equal to summation of Eqs. (A3) and (A4), which by rearranging terms results in the master equation, Eq. (10).

\section{APPENDIX B: GAUSSIAN EVOLUTION}

Here we briefly review the description of multimode Gaussian bosonic states and their open systems' evolution. Consider a system composed of $N$ bosonic modes. We work in the basis of Hermitian position and momentum operators for each mode, $\hat{q}=\frac{1}{\sqrt{2}}\left(\hat{a}+\hat{a}^{\dagger}\right)$ and $\hat{p}=\frac{1}{i \sqrt{2}}\left(\hat{a}-\hat{a}^{\dagger}\right)$, and the canonical commutation relations are $\left[\hat{q}_{\ell}, \hat{p}_{m}\right]=\delta_{\ell m}$. Noting the commutation relation for vectors of operators, $\left[\hat{\mathbf{r}}, \hat{\mathbf{s}}^{\top}\right]=$ $\hat{\mathbf{r}} \hat{\mathbf{S}}^{\top}-\left(\hat{\mathbf{s}} \hat{\mathbf{r}}^{\top}\right)^{\top}$, where ${ }^{\top}$ denotes matrix transpose, we define a column vector of stacked position and momentum operators, $\hat{\mathbf{x}}:=(\hat{\mathbf{q}} \hat{\mathbf{p}})^{\top}$. Then, the canonical commutation relations can be succinctly stated as

$$
\left[\hat{\mathbf{x}}, \hat{\mathbf{x}}^{\top}\right]=i \boldsymbol{\Omega}
$$

where the matrix $\boldsymbol{\Omega}$ is called the symplectic form and has the following representation in the $q p$ basis:

$$
\boldsymbol{\Omega}=\left(\begin{array}{cc}
\mathbf{0} & \mathbf{I} \\
-\mathbf{I} & \mathbf{0}
\end{array}\right),
$$

with $\mathbf{I}$ being the $N \times N$ identity matrix.

A Gaussian quantum state of $N$ bosonic modes is entirely described by a vector of means $\vec{x}:=\langle\hat{\mathbf{x}}\rangle$ and a symmetrized covariance matrix $\Sigma$ with elements $\Sigma_{i j}=\frac{1}{2}\left\langle\hat{x}_{i} \hat{x}_{j}+\hat{x}_{j} \hat{x}_{i}\right\rangle$. The covariance matrix for the vacuum state is $\boldsymbol{\Sigma}_{0}=\frac{1}{2} \mathbf{I}$. The squeezing operator in Eq. (22) was defined with respect to its Schrödinger-picture action; to find the covariance matrix we need its Heisenberg-picture action. Using $\hat{S}^{\dagger}(r, \vartheta)=\hat{S}(r, \vartheta+$ $\pi)$, the covariance matrix for a two-mode squeezed state can be found from its associated symplectic matrix [62],

$$
\Sigma(r)=\frac{1}{2}\left(\begin{array}{cccc}
\cosh (2 r) & -\sinh (2 r) & 0 & 0 \\
-\sinh (2 r) & \cosh (2 r) & 0 & 0 \\
0 & 0 & \cosh (2 r) & \sinh (2 r) \\
0 & 0 & \sinh (2 r) & \cosh (2 r)
\end{array}\right) \text {, }
$$

where we set $\vartheta=0$ for simplicity.

Gaussianity of a quantum state is preserved under evolution generated by Hamiltonians quadratic in the mode operators and jump operators that are linear in the mode operatorsthe master equation in Eq. (10) is one such example. Given a Lindblad master equation with $M$ jump operators that governs the evolution of a multimode bosonic state,

$$
\dot{\hat{\rho}}=-i[\hat{H}, \hat{\rho}]+\sum_{m=1}^{M} \mathcal{D}\left[\hat{L}_{m}\right] \hat{\rho},
$$

we may translate this into an evolution for the means and the covariance matrix without loss of information if Gaussianity is preserved. The Gaussian-preserving conditions require that the Hamiltonian can be written as

$$
\hat{H}=\frac{1}{2} \hat{\mathbf{x}}^{\top} \mathbf{G} \hat{\mathbf{x}},
$$

expressed in terms of the symmetric, real matrix $\mathbf{G} \in \mathbb{R}^{2 N \times 2 N}$, and each jump operator has the form

$$
\hat{L}_{m}=\sqrt{\gamma_{m}} \sum_{\ell=1}^{N}\left(Q_{m \ell} \hat{q}_{\ell}+P_{m \ell} \hat{p}_{\ell}\right),
$$

where $\gamma_{m}$ is the associated dissipation rate. Collecting the $Q_{m \ell}$ and $P_{m \ell}$ coefficients into the matrices $\mathbf{Q}$ and $\mathbf{P}$, the vector of $M$ jump operators is

$$
\hat{\mathbf{L}}=\mathbf{C} \hat{\mathbf{x}}
$$

with $\mathbf{C}:=(\mathbf{Q} \mathbf{P}) \in \mathbb{C}^{M \times 2 N}$. The means and covariance matrix obey the following equations of motion [84]:

$$
\begin{gathered}
\dot{\vec{x}}=\mathbf{A} \vec{x}, \\
\dot{\mathbf{\Sigma}}=\mathbf{A} \boldsymbol{\Sigma}+\mathbf{\Sigma} \mathbf{A}^{\top}+\mathbf{B},
\end{gathered}
$$

with matrices

$$
\begin{gathered}
\mathbf{A}=\boldsymbol{\Omega}\left(\mathbf{G}+\operatorname{Im}\left[\mathbf{C}^{\mathrm{H}} \mathbf{C}\right]\right), \\
\mathbf{B}=\boldsymbol{\Omega} \operatorname{Re}\left[\mathbf{C}^{\mathrm{H}} \mathbf{C}\right] \boldsymbol{\Omega}^{\top},
\end{gathered}
$$

where ${ }^{\mathrm{H}}$ indicates matrix conjugate transpose (to distinguish it from the Hermitian adjoint ${ }^{\dagger}$ of an operator). For the two-mode $\mathrm{ME}$ in Sec. IV $\mathbf{G}=\mathbf{0}$ and the jump operators are given by Eq. (20). Setting $\vartheta=0$ the matrices in the covariance matrix evolution, Eq. (B9), are $\mathbf{A}=\frac{\Gamma}{2} \mathbf{I}$ and $\mathbf{B}=\Gamma \boldsymbol{\Sigma}(r)$.

The Uhlmann-Jozsa fidelity

$$
\mathcal{F}\left(\hat{\rho}_{1}, \hat{\rho}_{2}\right)=\left[\operatorname{tr}\left(\sqrt{\sqrt{\hat{\rho}_{1}} \hat{\rho}_{2} \sqrt{\hat{\rho}_{1}}}\right)\right]^{2}
$$

is a measure of the closeness of the quantum states $\hat{\rho}_{1}$ and $\hat{\rho}_{2}$. When both states are Gaussian and at least one is pure, the fidelity can be calculated directly from their respective covariances matrices, $\boldsymbol{\Sigma}_{\rho_{1}}$ and $\boldsymbol{\Sigma}_{\rho_{2}}$,

$$
\mathcal{F}\left(\hat{\rho}_{1}, \hat{\rho}_{2}\right)=\left[\operatorname{det}\left(\boldsymbol{\Sigma}_{\rho_{1}}+\boldsymbol{\Sigma}_{\rho_{2}}\right)\right]^{-1 / 2},
$$

where $\operatorname{det}(\mathbf{A})$ is the determinant of the matrix $\mathbf{A}$ [85]. In Sec. IV the two-mode squeezed state is pure with a covariance matrix given by Eq. (B3).
[1] S. M. Barnett et al., Prog. Quantum Electron. 54, 19 (2017).

[2] M. Müller et al., Engineered open systems and quantum simulations with atoms and ions, in Advances In Atomic, Molecular, and Optical Physics (Academic Press, New York, 2012), Vol. 61, pp. 1-80.
[3] F. Verstraete, M. M. Wolf, and J. I. Cirac, Nat. Phys. 5, 633 EP (2009)

[4] F. Pastawski, L. Clemente, and J. I. Cirac, Phys. Rev. A 83, 012304 (2011).

[5] Z. Leghtas et al., Science 347, 853 (2015). 
[6] Y. Lin et al., Nature (London) 504, 415 EP (2013).

[7] S. Shankar et al., Nature (London) 504, 419 (2013).

[8] D. Kienzler et al., Science 347, 53 (2014).

[9] M. E. Kimchi-Schwartz, L. Martin, E. Flurin, C. Aron, M. Kulkarni, H. E. Tureci, and I. Siddiqi, Phys. Rev. Lett. 116, 240503 (2016).

[10] B. Q. Baragiola and J. Twamley, New J. Phys. 20, 073029 (2018).

[11] H. J. Carmichael, Statistical Methods in Quantum Optics (Springer-Verlag, Berlin, 2008).

[12] H. M. Wiseman and G. J. Milburn, Quantum Measurement and Control (Cambridge University Press, Cambridge, UK, 2010).

[13] H.-P. Breuer and F. Petruccione, The Theory of Open Quantum Systems (Oxford University Press, Oxford, 2003).

[14] T. A. Brun, Am. J. Phys. 70, 719 (2002).

[15] S. Attal and Y. Pautrat, Ann. Henri Poincaré 526, 59 (2006).

[16] S. Attal and A. Joye, J. Stat. Phys. 126, 1241 (2007).

[17] L. Bruneau, A. Joye, and M. Merkli, J. Math. Phys. 55, 075204 (2014).

[18] J. Rau, Phys. Rev. 129, 1880 (1963).

[19] V. Scarani, M. Ziman, P. Štelmachovič, N. Gisin, and V. Bužek, Phys. Rev. Lett. 88, 097905 (2002).

[20] V. Giovannetti and G. M. Palma, Phys. Rev. Lett. 108, 040401 (2012).

[21] S. Lorenzo, F. Ciccarello, and G. M. Palma, Phys. Rev. A 96, 032107 (2017).

[22] F. Ciccarello, Quantum Meas. Quantum Metrol. 4, 53 (2017).

[23] D. Layden, E. Martín-Martínez, and A. Kempf, Phys. Rev. A 93, 040301(R) (2016).

[24] D. Grimmer, D. Layden, R. B. Mann, and E. Martín-Martínez, Phys. Rev. A 94, 032126 (2016).

[25] D. Grimmer, R. B. Mann, and E. Martín-Martínez, Phys. Rev. A 95, 042114 (2017).

[26] N. Altamirano, P. Corona-Ugalde, R. B. Mann, and M. Zych, New J. Phys. 19, 013035 (2017).

[27] J. M. Horowitz, Phys. Rev. E 85, 031110 (2012).

[28] S. Lorenzo, R. McCloskey, F. Ciccarello, M. Paternostro, and G. M. Palma, Phys. Rev. Lett. 115, 120403 (2015).

[29] C. B. Dağ, W. Niedenzu, Ö. E. Müstecaplioğlu, and G. Kurizki, Entropy 18, 244 (2016).

[30] P. Strasberg, G. Schaller, T. Brandes, and M. Esposito, Phys. Rev. X 7, 021003 (2017).

[31] L. Li, J. Zou, H. Li, B.-M. Xu, Y.-M. Wang, and B. Shao, Phys. Rev. E 97, 022111 (2018).

[32] J. A. Gross, C. M. Caves, G. J. Milburn, and J. Combes, Quantum Sci. Technol. 3, 024005 (2017).

[33] Á. Cuevas, A. Geraldi, C. Liorni, L. D. Bonavena, A. De Pasquale, F. Sciarrino, V. Giovannetti, P. Mataloni, arXiv:1809.01922.

[34] A. N. Korotkov, Phys. Rev. A 65, 052304 (2002).

[35] T. Rybár, S. N. Filippov, M. Ziman, and V. Bužek, J. Phys. B 45, 154006 (2012).

[36] S. Kretschmer, K. Luoma, and W. T. Strunz, Phys. Rev. A 94, 012106 (2016).

[37] S. Campbell, F. Ciccarello, G. M. Palma, and B. Vacchini, Phys. Rev. A 98, 012142 (2018).

[38] A. Dabrowska, G. Sarbicki, and D. Chruściński, Phys. Rev. A 96, 053819 (2017).

[39] K.-D. Wu, Z. Hou, Y.-Y. Zhao, G.-Y. Xiang, C.-F. Li, G.-C. Guo, J. Ma, Q.-Y. He, J. Thomp- son, and M. Gu, Phys. Rev. Lett. 121, 050401 (2018).

[40] A. Černoch, K. Bartkiewicz, K. Lemr, and J. Soubusta, Phys. Rev. A 97, 042305 (2018).

[41] R. Riedinger et al., Nature (London) 556, 473 (2018).

[42] C. F. Ockeloen-Korppi et al., Nature (London) 556, 478 (2018).

[43] P. Kurpiers et al., Nature (London) 558, 267 (2018).

[44] Y. S. Weinstein, Phys. Rev. A 82, 032326 (2010).

[45] S. M. Hashemi Rafsanjani, M. Huber, C. J. Broadbent, and H. H. Eberly, Phys. Rev. A 86, 062303 (2012).

[46] C. Pellegrini, Ann. Probab. 36, 2332 (2008).

[47] G. Nogues et al., Nature (London) 400, 239 (1999).

[48] J. E. Gough, M. R. James, and H. I. Nurdin, in 50th IEEE Conference on Decision and Control and European Control (IEEE, New York, 2011), pp. 5570-5576.

[49] B. Q. Baragiola, R. L. Cook, A. M. Brańczyk, and J. Combes, Phys. Rev. A 86, 013811 (2012).

[50] B. Q. Baragiola and J. Combes, Phys. Rev. A 96, 023819 (2017).

[51] A. Dąbrowska, G. Sarbicki, and D. Chruściński, arXiv:1808.07398.

[52] F. Barra, Sci. Rep. 5, 14873 (2015).

[53] G. De Chiara, G. Landi, A. Hewgill, B. Reid, A. Ferraro, A. J. Roncaglia, M. Antezza, New J. Phys. 20, 113024 (2018).

[54] C. Sayrin et al., Nature (London) 477, 73 EP (2011).

[55] P. Campagne-Ibarcq et al., Phys. Rev. Lett. 120, 200501 (2018).

[56] N. Didier, J. Guillaud, S. Shankar, and M. Mirrahimi, Phys. Rev. A 98, 012329 (2018).

[57] Y. Y. Jau, A. M. Hankin, T. Keating, I. H. Deutsch, and G. W. Biedermann, Nat. Phys. 12, 71 (2016).

[58] K. M. Maller, M. T. Lichtman, T. Xia, Y. Sun, M. J. Piotrowicz, A. W. Carr, L. Isenhower, and M. Saffman, Phys. Rev. A 92, 022336 (2015).

[59] X.-B. Wang, T. Hiroshima, A. Tomita, and M. Hayashi, Phys. Rep. 448, 1 (2007).

[60] R. Schnabel, Phys. Rep. 684, 1 (2017).

[61] M. Gu, C. Weedbrook, N. C. Menicucci, T. C. Ralph, and P. van Loock, Phys. Rev. A 79, 062318 (2009).

[62] N. C. Menicucci, S. T. Flammia, and P. van Loock, Phys. Rev. A 83, 042335 (2011).

[63] C. Eichler, D. Bozyigit, C. Lang, M. Baur, L. Steffen, J. M. Fink, S. Filipp, and A. Wallraff, Phys. Rev. Lett. 107, 113601 (2011).

[64] T. Eberle, V. Händchen, and R. Schnabel, Opt. Express 21, 11546 (2013).

[65] C. F. Ockeloen-Korppi, E. Damskägg, J.-M. Pirkkalainen, T. T. Heikkilä, F. Massel, and M. A. Sillanpää, Phys. Rev. Lett. 118, 103601 (2017).

[66] E. Flurin, N. Roch, F. Mallet, M. H. Devoret, and B. Huard, Phys. Rev. Lett. 109, 183901 (2012).

[67] Z. Miao and A. Sarlette, Quantum Sci. Technol. 2, 034013 (2017).

[68] F. Benatti, R. Floreanini, and U. Marzolino, Europhys. Lett. 88, 20011 (2009).

[69] L. Mazzola, S. Maniscalco, J. Piilo, K.-A. Suominen, and B. M. Garraway, Phys. Rev. A 79, 042302 (2009).

[70] F. Benatti, R. Floreanini, and U. Marzolino, Phys. Rev. A 81, 012105 (2010).

[71] B. Bucča and T. Prosen, New J. Phys. 14, 073007 (2012).

[72] D. Manzano and P. I. Hurtado, Phys. Rev. B 90, 125138 (2014). 
[73] V. V. Albert and L. Jiang, Phys. Rev. A 89, 022118 (2014).

[74] A. Peres, Phys. Rev. Lett. 77, 3264 (1996).

[75] M. B. Plenio, Phys. Rev. Lett. 95, 090503 (2005).

[76] R. F. Werner, Phys. Rev. A 40, 4277 (1989).

[77] D. M. Greenberger, M. A. Horne, and A. Zeilinger, arXiv:0712.0921.

[78] J. T. Barreiro et al., Nat. Phys. 9, 559 (2013).

[79] J. Sperling and W. Vogel, Phys. Rev. Lett. 111, 110503 (2013).
[80] L. Pezzè, Y. Li, W. Li, and A. Smerzi, PNAS 113, 11459 (2016). [81] D. Girolami and B. Yadin, Entropy 19, 124 (2017).

[82] M. Ghio et al., J. Phys. A 51, 045302 (2018).

[83] D. Boyanovsky and D. Jasnow, Phys. Rev. A 96, 012103 (2017).

[84] K. Koga and N. Yamamoto, Phys. Rev. A 85, 022103 (2012).

[85] G. Spedalieri, C. Weedbrook, and S. Pirandola, J. Phys. A 46, 025304 (2013). 